\title{
9 The Upside-Down Jellyfish Cassiopea xamachana as an Emerging Model System to Study Cnidarian-Algal Symbiosis
}

\author{
Mónica Medina, Victoria Sharp, Aki Ohdera, Anthony Bellantuono, \\ Justin Dalrymple, Edgar Gamero-Mora, Bailey Steinworth, \\ Dietrich K. Hofmann, Mark Q. Martindale, André C. Morandini, \\ Matthew DeGennaro and William K. Fitt
}

\section{CONTENTS}

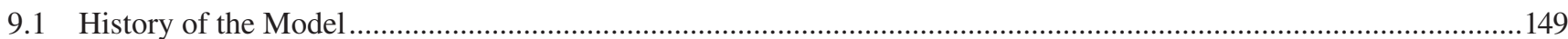

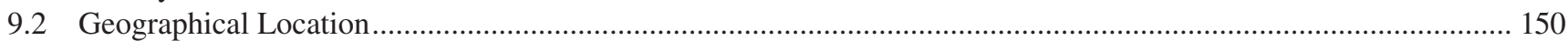

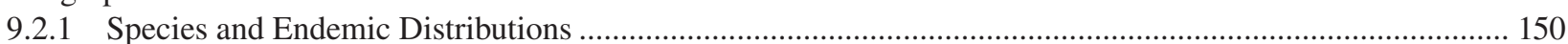

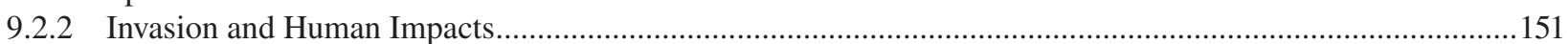

9.3 Life Cycle

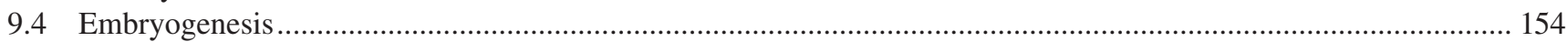

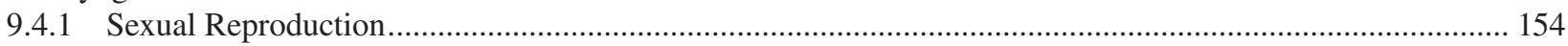

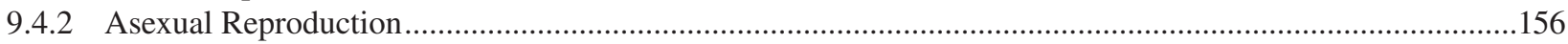

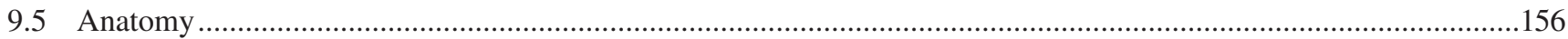

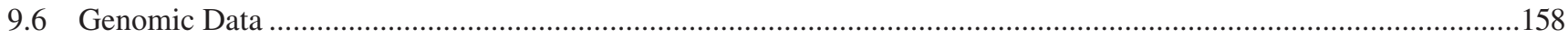

9.7 Functional Approaches: Tools for Molecular and Cellular Analyses......................................................................160

9.7.1 Toward a Genetic Model to Study Cnidarian Symbiosis ...........................................................................

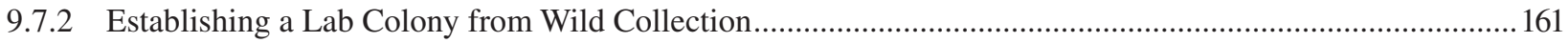

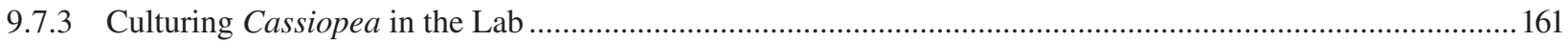

9.7.4 Microinjection of Single-Cell Embryos for the Generations of Mutants and Transgenic Cassiopea .............163

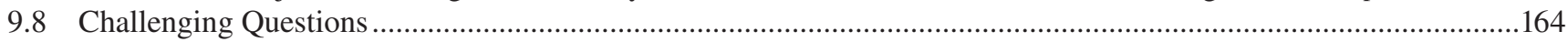

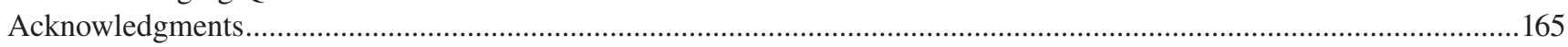

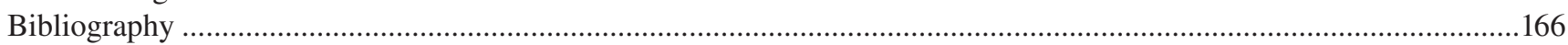

\subsection{HISTORY OF THE MODEL}

The model Cassiopea xamachana, also known as the upside-down jellyfish, was first described for the Caribbean (Jamaica) by Bigelow in 1892. Cassiopea xamachana is a tropical species belonging to the cnidarian class Scyphozoa, order Rhizostomeae, family Cassiopeidae. Substantially different from typically pelagic scyphozoan medusae, Cassiopea spp. jellyfish show an epibenthic lifestyle, resting upside-down with the bell turned to the substrate and the oral arms and appendages exposed upward. They preferentially occur in shallow water on soft bottom areas, often also in seagrass beds, in tropical, mangrove-sheltered lagoons.

Historically, Peter S. Pallas published the first formal description of a rhizostome medusa termed Medusa (now
Cassiopea) frondosa in 1774 , based on a preserved specimen originating from an unreported site in the Caribbean. However, Peter Forskål, a member of a Danish expedition sent to explore Arab countries in the years 1761-1767, first observed, collected and described in his data log an upside-down-type rhizostomatous medusa under the name Medusa (now Cassiopea) andromeda at Tôr on the southwestern coast of the Sinai Peninsula in October 1762. Tragically, Forskål and all but one participant of the expedition succumbed to disease or fatal incidents. As the only survivor, the surveyor Carsten Nibuhr wrote an account of the expedition and published postum only in 1775 the scientific descriptions of plants and animals Forskål had left behind. The plates depicting the described C. andromeda specimen were published a year later in 1776. Several 
more forms of Cassiopea medusae have been described from various tropical regions of the world by 19th-century authors, either as varieties of $C$. andromeda or as separate species and varieties thereof. These descriptions were compiled and critically reviewed by Mayer (1910). For an actual listing of valid Cassiopea species, see Ohdera et al. (2018) and Jarms and Morandini (2019). Cassiopea spp. have been recorded as alien or introduced species first in the Mediterranean Sea by Maas (1903), as so-called "Lessepsian migrants" originating from the Red Sea through the Suez Canal, and in O'ahu, Hawaii, described by Cutress in Doty (1961) as most probably introduced during World War II.

In his keystone paper, Bigelow (1892) provided a detailed description of the anatomy and development of $C$. xamachana from Jamaica bearing on both the medusa and the scyphopolyp (scyphistoma). He included medusa formation by strobilation of the polyp and the asexual propagation of the polyp through the budding of ciliated, spindle-shaped propagules that settle and develop into new polyps. Sexual reproduction by the typically gonochoric medusae was assessed much later and embryonic development approached only recently (see Section 9.4). Bigelow was a pioneer in noticing the presence of green cells, or "zoanthelae", in medusae, scyphistomae and buds of this species, recognized as symbiotic unicellular algae and described much later by Freudenthal (1959). They became commonly termed "zooxanthellae". A wealth of information on $C$. andromeda from the Red Sea became available through the two monographs by Gohar and Eisawy (1960a, 1960b), closing gaps in knowledge of the life-history. In contrast, information on C. frondosa remained scarce (Bigelow 1893; Smith 1936; Hummelinck 1968). Providing easily collectable mature medusae from tropical and subtropical habitats almost year-round, and with scyphistomae performing asexual reproduction under relatively simple conditions in the lab, C. xamachana was setting out to become a versatile symbiotic scyphozoan model species.

The Carnegie Marine Biological Laboratory on Loggerhead Key in the Dry Tortugas, Gulf of Mexico, commonly called Tortugas Marine Laboratory, was founded in 1904 with Alfred Goldsborough Mayer as its first director (Stephens and Calder 2006). This lab, in fortunate association with the publication series Papers from Tortugas Laboratory by the Carnegie Institution, was pivotal in hosting experimental studies of Cassiopea spp. (Perkins 1908). Some of the research topics included Cassiopea's rhythmical pulsation and its causes (Mayer 1908), the rate of regeneration in C. xamachana medusae (Stockard 1908), the physiology of the C. xamachana nervous system (Cary 1917) and the anatomy and physiology of the sympatric C. frondosa (Smith 1936). Mayer (1910) contributed volume III, The Scyphomedusae, of his monumental work, Medusae of the World. In it, he provides a detailed account of the genera Toreuma and Cassiopea in the context of history, taxonomy and biology. After those early 20th-century works, there was a slowdown in research in Cassiopea, with a renaissance in the 1970s. Curtis and Cowden (1972) meticulously investigated the significant regenerative capacities of $C$. xamachana scyphistomae. More recently, Hamlet et al. (2011) and Santhanakrishnan et al. (2012) introduced advanced high speed kinematic and modeling techniques to study the hydrodynamics of the conspicuous pulsation behavior of the Cassiopea jellyfish. Moreover, in the wake of photo-physiological studies of zooxanthellate scleractinian corals (e.g. Yonge and Nicholls 1931), the CassiopeaSymbiodinium symbiosis prompted a rapidly growing number of studies bearing on the mutualistic relationship between the host and the algal symbionts in different phases of the life cycle (e.g. Ludwig 1969; Balderston and Claus 1969; Hofmann and Kremer 1981; Fitt and Trench 1983a). Contemporary work on bud-to-polyp transition by Curtis and Cowden (1971) initiated a search for extrinsic natural and synthetic factors inducing metamorphosis of planula larvae and buds and studies to elucidate their putative mode of action (see Section 9.3). In recent years, research on $C$. xamachana diversified considerably, as described in 2018 by Ohdera and a consort of co-authors. Their review exposes work on behavior, quiescence, bioinvasions and blooms, environmental monitoring and ecotoxicology, toxicology and cnidome and virology, in addition to expanding on topics that have briefly been considered here. The isolation of Hox genes by Kuhn et al. (1999) was a landmark timepoint indicating that $C$. xamachana research had entered the age of evo-devo and genomics (see Section 9.6).

\subsection{GEOGRAPHICAL LOCATION}

\subsubsection{Species and Endemic Distributions}

It is often the case that jellyfish clades include cryptic species not easily distinguished by morphological characteristics (Holland et al. 2004; Arai 2001), and this is further complicated by the fact that intraspecific morphological diversity is often quite high (Gomez-Daglio and Dawson 2017). Nine Cassiopea species are currently recognized by the World Register of Marine Species: $C$. andromeda (Forskål 1775), C. depressa (Haeckel 1880), C. frondosa (Pallas 1774), C. maremetens (Gershwin et al. 2010), C. medusa (Light 1914), C. mertensi (Brandt 1835), C. ndrosia (Agassiz and Mayer 1899), C. ornata (Haeckel 1880) and C. xamachana (Bigelow 1892). Additionally, C. vanderhorsti has been proposed as a species (Stiasny 1924) but may be a variety of $C$. xamachana (Jarms and Morandini 2019). Cassiopea species are distributed throughout tropical and subtropical waters all over the world, with $C$. frondosa and C. xamachana in the Caribbean and Gulf of Mexico; C. andromeda in the Red Sea, invasive in Hawaii, Brazil and the Asian-Australian sea; C. medusa, C. mertensi, $C$. maremetens, $C$. ndrosia and $C$. ornata in the eastern South Pacific; and $C$. depressa along the coral coast of eastern African in the Indian Ocean (Figure 9.1).

Morphological work would go on to merge C. medusa and C. mertensi into C. andromeda (Gohar and Eisawy 1960a) 


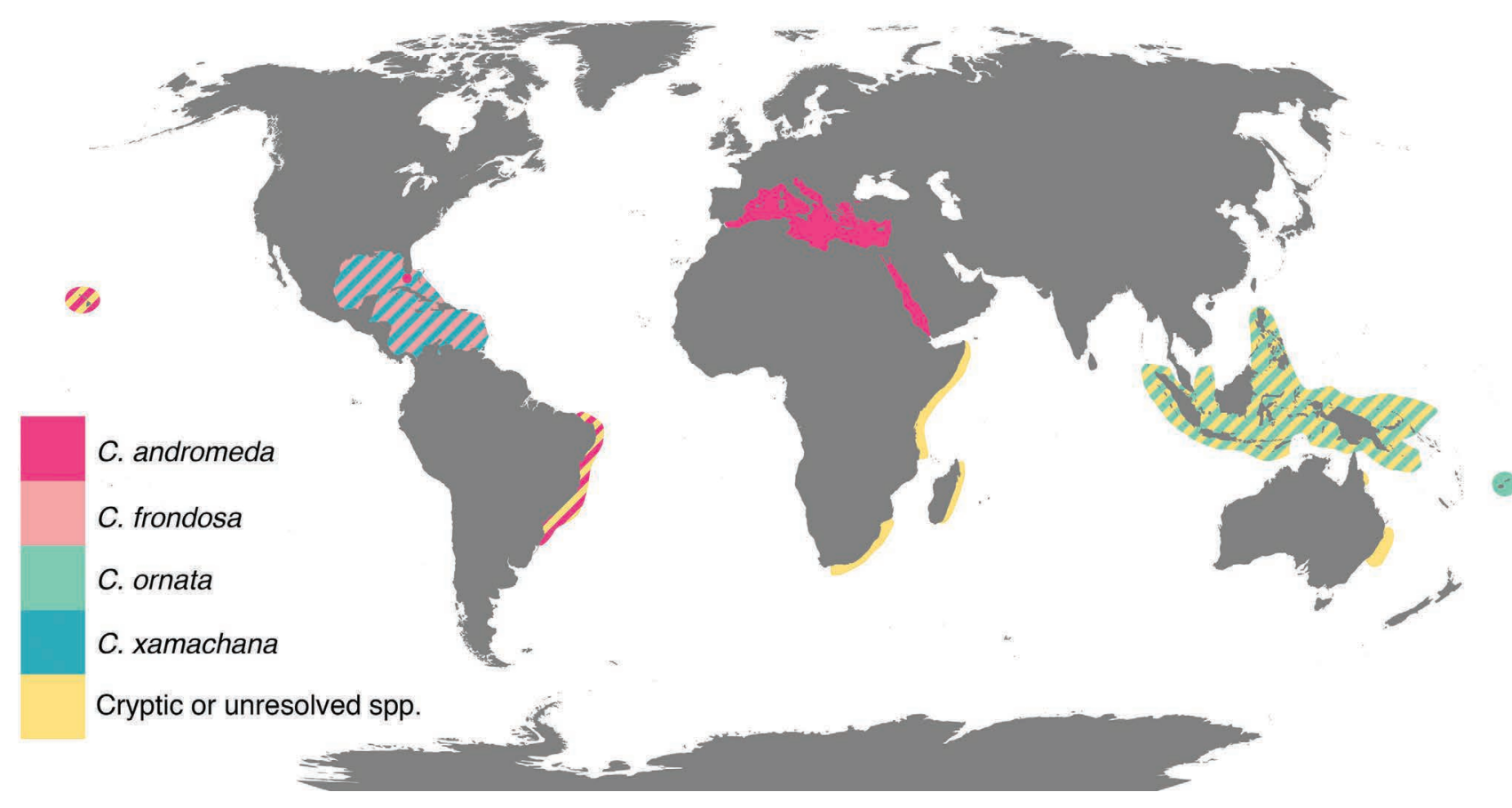

FIGURE 9.1 Estimated global distribution of Cassiopea species, compiled from the World Register of Marine Species. (From Holland et al. 2004, Arai et al. 2017, and Morandini et al. 2017.)

before further reorganization of the clade by molecular phylogenetic analysis. In recent years, several groups have used DNA barcoding of the mitochondrial gene cytochrome c oxidase subunit 1 (usually denoted as COI or COX1) to resolve ambiguities in the phylogeny of Cassiopea. Analysis of COX1 sequences from Cassiopea around the world by Holland et al. (2004) supports six species: C. frondosa in the western Atlantic; $C$. andromeda in the Red Sea, western Atlantic and Hawaii; C. ornata in Indonesia, Palau and Fiji; cryptic Cassiopea species 1 in eastern Australia; cryptic Cassiopea species 2 in Papua New Guinea; and cryptic Cassiopea species 3 in Papua New Guinea and Hawaii. The three cryptic species suggested by this analysis were previously classified as $C$. andromeda. This study also shows that specimens identified as C. xamachana from the Gulf of Mexico and the Caribbean are actually $C$. andromeda. Later studies by Morandini et al. (2017) and Arai et al. (2017) largely recapitulate these findings, but Arai et al. (2017) suggest three more cryptic species within $C$. andromeda, potentially bringing the total number of Cassiopea species to as many as nine, plus the valid morphospecies without molecular data associated with them (C. depressa, $C$. maremetens, C. medusa, C. mertensi and C. ndrosia). Further work remains to be done in this field, especially considering the claim that COX1 barcoding may be insufficient to distinguish between cnidarian congeners due to exceptionally low rates of mitochondrial evolution within Cnidaria (France and Hoover 2002; Shearer et al. 2002). This is possibly due to the presence of excision repair, which is absent in other animal mitochondria (Hebert et al. 2003).

\subsubsection{INVASION AND HUMAN IMPACTS}

Cassiopea jellyfish possess multiple characteristics which make them a potential invasive threat, particularly their high tolerance to both salinity (Goldfarb 1914) and thermal stress (Klein et al. 2019), as well as their capacity for thermal acclimation to $32^{\circ} \mathrm{C}$ (Al-jbour et al. 2017). Recent work suggests that rising seawater temperatures may increase the range of Cassiopea (Al-jbour et al. 2017). With cryptic life phases and potential to persist as scyphistomae (= benthic stages) for extended periods of time, Cassiopea have great potential to be transported as hitchhikers on ships. Additionally, proximity to human populations may enhance Cassiopea growth: there is some evidence from Abaco Island (Bahamas) that Cassiopea populations are larger in areas with high human density, presumably since high human densities are also correlated with higher levels of nutrients (Stoner et al. 2011; Thé et al. 2020).

The potential for Cassiopea invasion and blooms has been realized in multiple instances. Humans have a historical role in spreading Cassiopea, with molecular evidence suggesting that Floridian and Bermudan Cassiopea were spread to Brazil approximately 500 years ago-a time contemporaneous with the beginning of Portuguese shipping and colonization in the region (Morandini et al. 2017).

The relationship between human movement and Cassiopea range extension has also been documented more recently. The Hawaiian Islands have apparently been colonized by Cassiopea in the past century, as a 1902 survey by Mayer (1906) on the USS Albatross, the first purpose-built marine research ship, found no Cassiopea on the islands. Cassiopea 
were first reported after World War II, presumably transported to Hawaii by US naval traffic. According to reports by residents, Cassiopea medusa first appeared exclusively in Pearl Harbor on O'ahu between 1941 and 1945 but were observed circa 1950 in Honolulu Harbor and the Ala Wai Canal (Doty 1961). Observations in 1964 (Uchida 1970) reported Cassiopea in Kane'ohe Bay. These early reports of Cassiopea initially identified C. medusa and C. mertensi, but the taxa have since been collapsed to a single species, Cassiopea andromeda, due to morphological similarity (Hofmann and Hadfield 2002). Curiously, however, the Cassiopea found near Ala Wai Harbor exhibited hermaphroditism, though this characteristic was not stable over time (Hofmann and Hadfield 2002).

Baker's law (1955) hypothesizes that species which can reproduce with only a single hermaphroditic parent will colonize new areas more successfully than gonochoristic species. While the advantages in invasion capacity of uniparental reproduction have not been tested in cnidarians, this ability is the basis of a longstanding hypothesis in terrestrial plants (Baker 1965; Van Etten et al. 2017). The hermaphroditic capacity of some Cassiopea may facilitate their invasion, particularly of islands seeded by chance through human introduction, where a founding population may originate from a single scyphistoma hitchhiking on a hull or in ballast water. Indeed, Hofmann and Hadfield (2002) hypothesize that the founder of the invasive population in Ala Wai Canal may have consisted of a single clonal individual. Morandini et al. (2017) note that all 200 medusae collected in Cabo Frio (Brazil) were male and potentially the result of clonal reproduction, suggesting that asexual reproduction as scyphistomae is yet another method of uniparental reproduction that may play a part in the capacity of Cassiopea to expand their range. A recent study from northeastern Brazil (Ceará state) also reported only female individuals in the population (Thé et al. 2020).

The first molecular phylogenetics of Cassiopea indicated that the species identified as C. andromeda in O'ahu, Hawaii, waters in fact comprised two distinct clades representing a cryptic species (Holland et al. 2004), with one clade of Indo-Pacific origin and the other established from either the Western Atlantic or Red Sea. Arai et al. (2017) further examined the molecular phylogenetics of Cassiopea and also found that $C$. xamachana from the Western Atlantic and $C$. andromeda from the Red Sea fell into the same clade, indicating that these are likely the result of an introduction of $C$. andromeda into the Caribbean.

Cassiopea have recently spread even farther, with reports in the central Mediterranean originally in 2005 in the Maltese Islands (Schembri et al. 2010) and again in 2006 in the Levantine coast of Turkey (Çevik et al. 2006). Keable and Ahyong (2016) identified multiple species in coastal lakes of eastern Australia, representing the southernmost reported invasion of the genus (Figure 9.1). The growing geographic range and propensity of Cassiopea to form blooms further supports the need for revised systematic and taxonomic methods for the accurate classification of these organisms in order to more meaningfully categorize them and identify their origins.

\subsection{LIFE CYCLE}

Like the majority of scyphozoans, C. xamachana alternates between the asexual polyp (i.e. scyphistoma) and a sexual medusa (Figure 9.2). Planula larvae, the result of sexual reproduction, settle and metamorphose in response to bacterial cues on environmental substrates (Hofmann et al. 1996) (for early development, see Section 9.4). The resulting scyphistomae can reproduce asexually via budding or strobilation to produce either a male or female medusa. Strobilation is initiated following the establishment of symbiosis with dinoflagellates of the family Symbiodiniaceae (LaJeunesse et al. 2018). Therefore, in addition to environmental factors, life cycle completion partly involves association with two different organisms: settlement of the larvae happens in response to different bacterial cues, and strobilation occurs in response to cues associated with the establishment of symbiosis with Symbiodiniaceae.

The planula larva does not have dinoflagellate symbionts but does rely on specific bacteria such as Vibrio spp. (Neumann 1979; Hofmann and Brand 1987) and Pseudoalteromonas sp. (Ohdera, et al., in prep a) that release cues to induce their settlement and metamorphosis. The cues appear to be peptides that are either released by the bacteria or the result of biodegradation of the substrate they are on (Fleck et al. 1999). A number of artificial peptides have been identified and the mechanism of interaction with larval receptors proposed (Hofmann et al. 1996; Fleck and Hofmann 1995). The scyphistomae are frequently found on the shaded side of degraded mangrove leaves during the summer (Fleck and Fitt 1999; Fleck et al. 1999) but also settle on other leaves and hard surfaces.

Newly settled scyphistomae of $C$. xamachana exhibit horizontal transfer of symbiotic Symbiodiniaceae, meaning they collect their symbionts from the environment rather than inheriting them. Shortly after settling and metamorphosing into polyps and developing a mouth, endodermal digestive cells (i.e. gastrodermis) phagocytose Symbiodiniaceae from the water column (Colley and Trench 1983). Soon after being infected with symbiotic algae, the scyphistoma undergoes strobilation. Algae live within the symbiosome, also known as the amoebocyte, formed from the initial vacuoles which engulf the ingested symbiont cells. Amoebocytes migrate to the base of the gastrodermis by approximately day 3 after ingestion and subsequently migrate to the mesoglea by approximately day 8 post-infection (Colley and Trench 1985). When the number of Symbiodiniaceae reach $5-12,000$ in large $(>1 \mathrm{~mm})$ scyphistomae at $\geq 25^{\circ} \mathrm{C}$, they will strobilate a single medusa in one to three weeks depending on temperature and light levels (Hofmann et al. 1978). We have observed that scyphistomae can continue strobilating throughout the summer and fall in the Florida Keys and in culture indefinitely. C. xamachana has been found to establish a symbiosis with different Symbiodiniaceae species in 


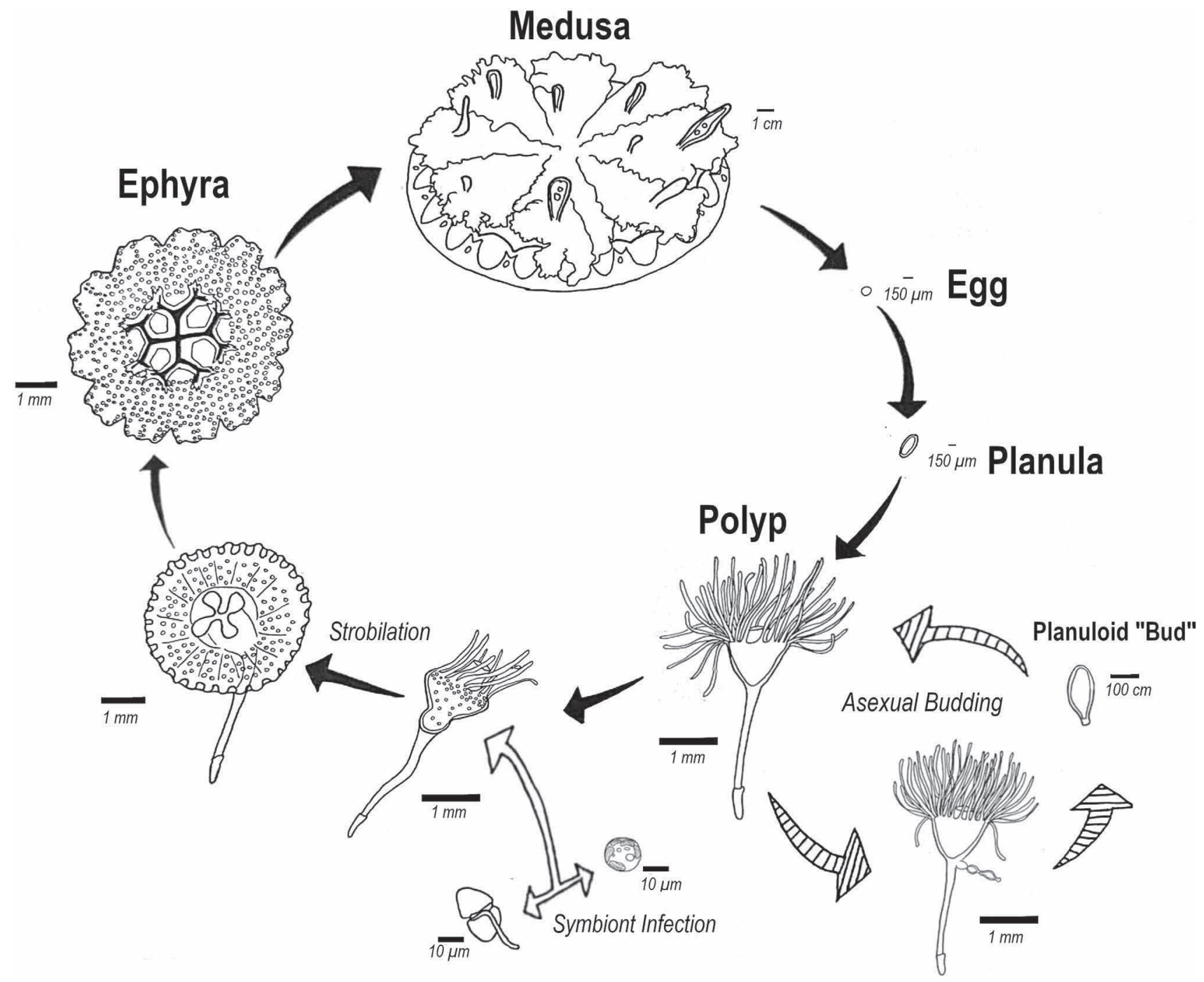

FIGURE 9.2 Life cycle of Cassiopea xamachana with scale bars per developmental stage. Ontogenetic stage names in bold. Nonsexual processes in italics. Black arrows; metagenic life cycle. Striped arrows; asexual "budding" reproduction. White arrows; symbiont infection and induction of strobilation.

fewer than three days while being held on the reef, back reef, seagrass bed or mangroves in the Florida Keys (Thornhill et al. 2006). If exposed to the homologous (found most frequently and at highest relative densities in C. xamachana) symbiont species Symbiodinium microadriaticum, the symbiont composition switches to Symbiodinium microadriaticum in a short period of time (via competitive exclusion), and the scyphistomae strobilates shortly thereafter (Thornhill et al. 2006). The role $S$. microadriaticum plays in inducing strobilation is not currently known.

The medusa and symbiotic scyphistomae are both photosynthetic and predatory. Photosynthesis occurs in the symbiotic dinoflagellates contained in digestive or ameobocytic cells, usually in direct sun in very shallow water, and is thought to provide the bulk of the fixed carbon to fulfill the energy requirements of their hosts (Verde and McCloskey 1998). However, they also use their mouth arm digitata, which contain the stinging organelles called nematocysts, to capture small zooplankton and other particles. Rhizostomes feed via many small mouths rather than the single mouth found in all other scyphozoans. C. xamachana can also shed clumps of nematocysts-dubbed cassiosomes-presumably to aid in obtaining food or as a defense from predators (Ames et al. 2020). External feeding is thought to provide the protein for growth of the jellyfish.

Temperature is a decisive factor in the life cycle of C. xamachana. Whereas rhizostome jellyfish typically over-winter in the scyphistomae stage, $C$. xamachana are present in the South Florida winter only as a medusa, as the polyps cannot feed themselves and disappear at temperatures $\leq 18^{\circ} \mathrm{C}$ (Fitt and Costley 1998). As the water temperature rises, planulae settle and metamorphose into scyphistomae which catch and consume food. It is not known if scyphistomae can survive winter temperatures in lower latitudes of the Caribbean Sea. C. xamachana begins to strobilate when temperatures are $\geq 25^{\circ} \mathrm{C}$, thus completing the life cycle (Rahat and Adar 1980). 
As temperatures increase with global climate change, populations of $C$. xamachana appear to be expanding (Morandini et al. 2005, Morandini lab unpublished) with a longer season to strobilate (Richardson et al. 2009). In addition, $C$. andromeda has become an exotic species, with populations in Australia, Hawaii, the Mediterranean and potentially the entire Caribbean (Çevik et al. 2006; Morandini et al. 2017; Holland et al. 2004; Schembri et al. 2010; Keable and Ahyong 2016), possibly partially due to higher temperatures. Whether the exotic $C$. xamachana's recent range expansions will harm the environment remains to be seen.

\subsection{EMBRYOGENESIS}

\subsubsection{Sexual Reproduction}

Members of the genus Cassiopea are generally gonochoristic, though hermaphrodites have been observed in at least one population (Hofmann and Hadfield 2002). In males, appendages are homogenous across the oral disc, whereas in females, there is a region of appendages at the center of the oral disc that are specialized for brooding embryos (circled in Figure 9.3a). The precise timing of sexual maturity is not known in terms of age or diameter; however, viable gametes have been recovered from individuals as small as $7 \mathrm{~cm}$ in bell diameter (Hofmann and Hadfield 2002). The gonads can be accessed through the four prominent openings (subgenital pits) located between the oral arms and the bell. In the Florida Keys, the temperatures are often colder during winter cold fronts, which could reduce the number of eggs female medusae produce.

Despite the existence of separate sexes, the site of fertilization is unknown. Free spawning has never been observed. Martin and Chia (1982) claim to have performed in vitro fertilization: they collected gonadal material from inside the gastrovascular cavity, combined ovary and testes in seawater and observed swimming planulae. Fertilization seems to occur either within the mother, with sperm taken in from the water column, or quickly after unfertilized eggs are deposited onto the brooding tentacles.

In laboratory conditions with adult wild-caught animals, new embryos can be collected daily from the brooding region of female medusae. Spawning seems to be regulated by light. When medusae are kept on a light cycle of 12 hours of darkness and 12 hours of light at $24^{\circ} \mathrm{C}$ at the Whitney Lab for Marine Bioscience, zygotes can be observed among the brooding appendages of female medusae, but only if male medusae are also present. If females are maintained separately from males, no eggs (fertilized or unfertilized) are observed to be released into the brooding appendage region. Unlike some symbiotic cnidarians, eggs do not contain symbiotic dinoflagellates; symbionts are acquired horizontally via acquisition from the environment rather than vertically inherited from the mother.

Within a few hours, clusters of zygotes become encased in a stiff membrane that attaches them firmly to the brooding tentacles (Figure 9.3b). This membrane is maternally produced, as zygotes collected from the mother before the membrane appears do not develop this membrane. Eggs have already been fertilized before this membrane appears. Embryos are tightly packed within this membrane, often causing them to take on irregular shapes as development progresses. If left undisturbed, zygotes will continue to develop encased in this membrane, attached to the mother's brooding appendages, until reaching the stage when they can swim using cilia and eventually free themselves and swim away.

Observations of development have been made from embryos removed at the one-cell stage and kept at $24^{\circ} \mathrm{C}$. Zygotes are 100-150 um in diameter (Figure 9.3c). Cleavage begins approximately two hours after zygotes are first observed (Figure 9.3d). Initial cell divisions are unipolar, beginning at the animal pole, and are complete, producing clear two-cell (Figure 9.3e) and four-cell (Figure 9.3f) stages. The embryo reaches the blastula stage, a hollow ball of cells with no yolk in the blastocoel, around 24 hours after the first cleavage (Figure 9.3g), and gastrulation is complete within 48 hours after the first cleavage is observed (Figure 9.3h). The exterior of the gastrula is ciliated (Figure 9.3k). Gastrulae move with a spinning motion, unlike the directed swimming later seen in the planula.

Further study is needed to fully understand the morphological details of development from zygote to planula. The mode of gastrulation is not yet known, though invagination is the most common form of gastrulation in the Scyphozoa (Morandini and da Silveira 2001; Nakanishi et al. 2008; Yuan et al. 2008; Kraus and Markov 2016). During gastrulation by invagination, the epithelium of the blastula folds inward at the future oral end while maintaining its epithelial identity. The epithelium continues to migrate inward until there are two layers of epithelium, the endoderm and ectoderm. Some cnidarians have complex patterns of gastrulation involving multiple waves of cellular movement (reviewed by Kraus and Markov 2016). While the mode of gastrulation has not been confirmed in Cassiopea, images of gastrulae appear to support the possibility of gastrulation by invagination (Figures 9.3h-i). Molecular studies using endomesodermal markers in other cnidarians are underway to confirm the location of presumptive endodermal precursors.

At three days old, an opening to the external sea water is still present and is located at the site of gastrulation, the blastopore (Figure 9.3i). By four days, the blastopore has closed completely, so that the inner epithelium has no connection to the outside of the embryo (Figure 9.3j). The structure of four-day-old planulae was described by Martin and Chia (1982) using transmission electron microscopy (TEM). Planulae range from 120 to $220 \mu \mathrm{m}$ in length and 85 to 100 $\mu \mathrm{m}$ in width at the midpoint. The exterior of the planula is uniformly ciliated (Figure 9.31), and planulae swim leading with the future aboral end ahead, but there is no apical tuft at the leading edge. Planulae contain endodermal and ectodermal epithelia separated by a thin layer of mesoglea (Martin and Chia 1982). 

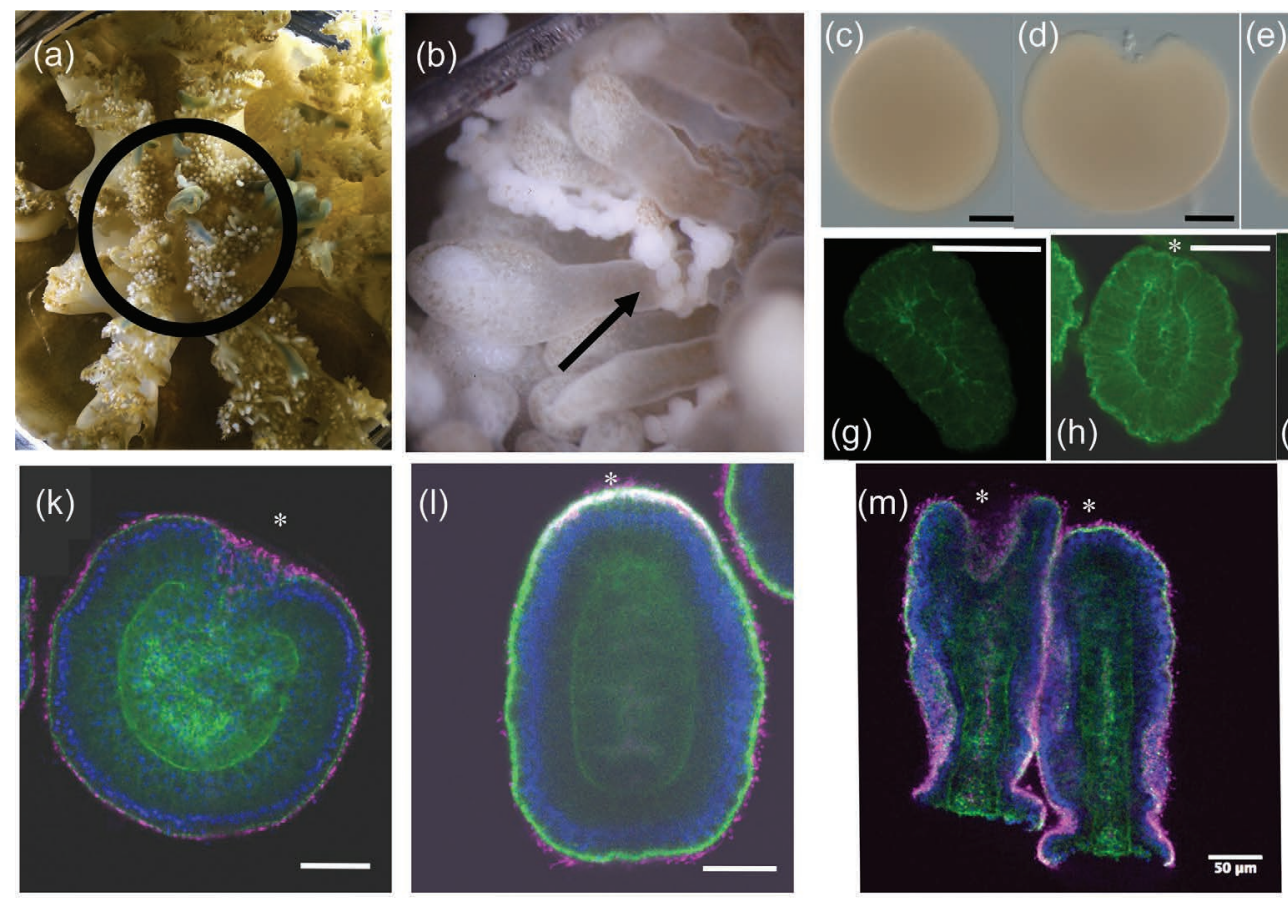

(i)
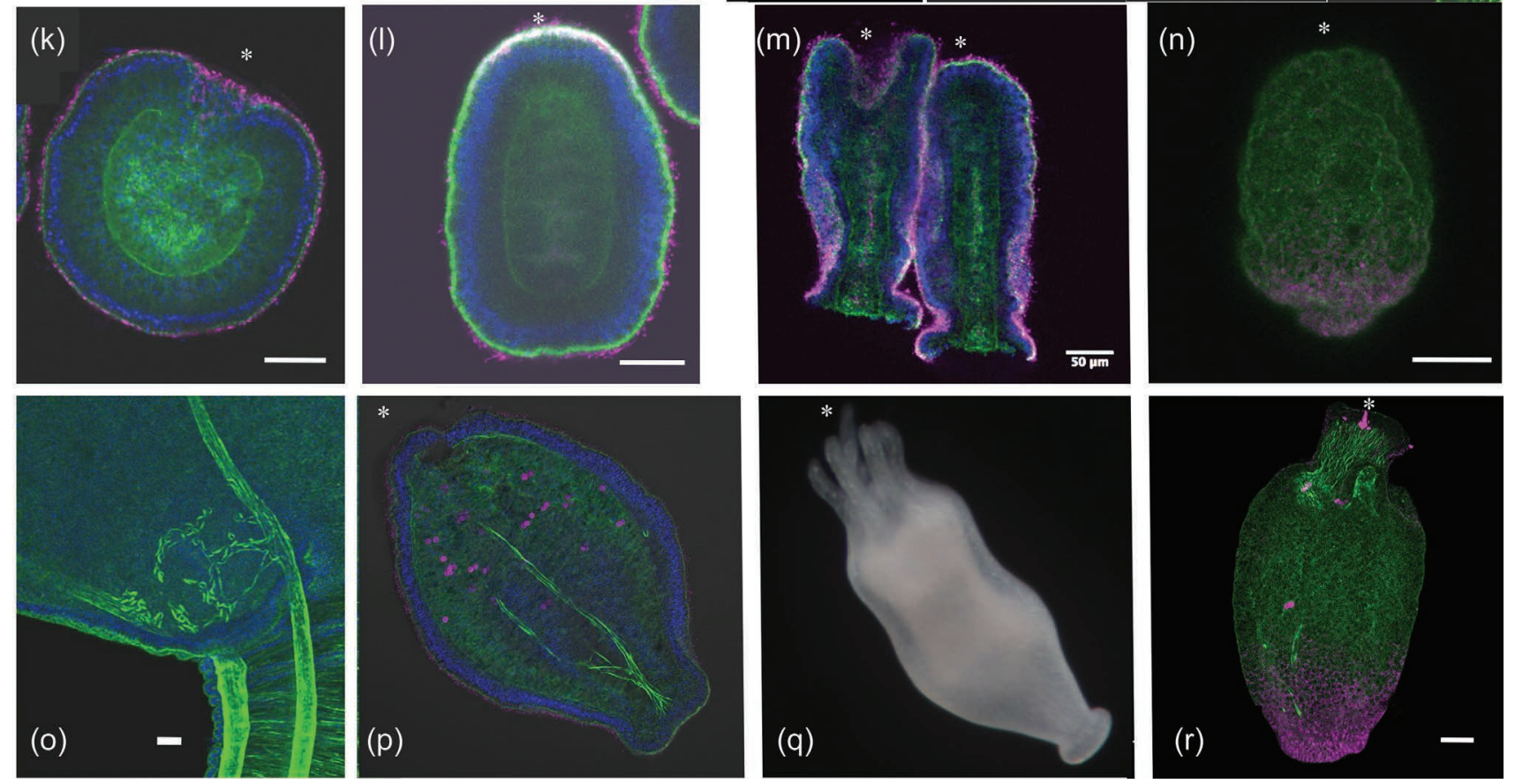

FIGURE 9.3 If female medusae (a) are kept with male medusae, zygotes can be found daily among the brooding appendages (b, circled in a) at the center of the oral disc. Zygotes (c) are packaged in a thin membrane and attached to the brooding appendages. Arrow in (b) points to attachment point where a package of embryos is wrapped around a brooding appendage. Location of fertilization is unknown. Initial cleavage (d) produces a two-cell stage (e), and each cell divides equally to produce a four-cell stage (f). Embryos reach the blastula stage (g) at approximately 24 hours after first cleavage and the gastrula stage (h) approximately 48 hours after first cleavage. At 72 hours after first cleavage (i), the blastopore can still be observed, but it is no longer observable by 96 hours (j). (g-j) Confocal slices stained to show actin. $(\mathrm{k}-\mathrm{l})$ and $(\mathrm{o}-\mathrm{p})$ Confocal slices stained to show actin (green), nuclei (blue), and cilia [magenta, no cilia stain in (o)]. Gastrulae (k) and planulae (l) are ciliated, and no mouth is observable in planulae. After attachment to a surface (m, right side), the polyp mouth forms de novo ( $\mathrm{m}$, left side). Asexually produced planuloids contain septal muscle fibers from the parent polyp (o and $\mathrm{p}$ ) and can contain symbiotic dinoflagellates in the gastrodermis, shown by magenta autofluorescence in (p). Mouth and tentacles can form in asexually produced planuloids without attachment to a substrate (q). Both planulae and asexually produced planuloids stain with antibodies to the neural marker protein RFamide ( $\mathrm{n}$ and $\mathrm{r}$ ), shown here on 3D projections of confocal stacks with RFamide in magenta and actin in green. All scale bars are 50 micrometers. Asterisks indicate the future oral end of planulae and planuloids.

Four cell morphologies have been previously described in the planula: two types in the ectoderm and two in the endoderm. The ectoderm consists of support cells and cnidocytes. Ectodermal support cells extend from the mesoglea to the exterior surface. The apical surface of a support cell is covered in microvilli, and each cell has a single cilium (Martin and Chia 1982). Martin and Chia report one type of cnidocyte in the planula but do not specify what type it is; in other life stages of Cassiopea, different types of cnidocytes have been described (Heins et al. 2015) (see Section 9.5 for additional detail). The apical surface of a mature cnidocyte is exposed to the exterior, and the cell does not appear to extend basally to the mesoglea, based on TEM. Developing cnidocytes can be identified by their capsule and are located between support cells near the basal region of these cells; they do not connect to the exterior. The endoderm also contains two cell types: support cells and interstitial cells. Endodermal support cells extend from the mesoglea to the interior lumen of the planula and bear an apical cilium. Interstitial cells are clustered among the endodermal support 
cells, and their function is unknown (Martin and Chia 1982). Staining with an antibody to the neurotransmitter RFamide implies the presence of neural cells, specifically concentrated at the aboral end of the planula. The potential presence of neural cells indicates there may be additional cell types present that have not yet been described.

Planulae are competent to settle by the age of four or five days (Martin and Chia 1982). Attachment to a surface usually precedes development into a polyp, but planulae have been observed to metamorphose without attachment (Martin and Chia 1982). Planula settlement can be induced by Vibrio alginolyticus bacteria or by the hexapeptide Z-Gly-Pro-GlyGly-Pro-Ala (Hofmann and Brand 1987). The polyp mouth forms de novo at the site of blastopore closure (Figure 9.3m), followed by four initial tentacles surrounding it, then four additional tentacles at the spaces between those. At this point, the former planula is recognizable as a small polyp. Once the mouth has developed, polyps are capable of both eating and taking in dinoflagellates from the environment to establish symbiosis. As the polyp grows, the region of the stolon that lacks a gastrovascular cavity continues to lengthen.

\subsubsection{Asexual Reproduction}

In addition to sexual reproduction, polyps can reproduce asexually to form more polyps. Clonal daughter offspring bud from the side of polyps, usually at consistent spots near the base of the calyx, in the form of swimming oblongs researchers have called planuloids or planuloid buds (Khabibulina and Starunov 2019). The future oral-aboral axis of the planuloid forms at an angle to the oral-aboral axis of the parent polyp. Clonal planuloids are superficially similar to planulae produced as a result of spawning in a number of ways. Both planulae and planuloids have a uniformly ciliated exterior; both swim leading with the future aboral end of the polyp ahead, rotating about the oral-aboral axis. An oral opening is absent in both (Figures 9.31 and p) and forms during development into a polyp (Figures $9.3 \mathrm{~m}$ and q). Additionally, antibody staining against the neural marker RFamide (Figures 9.3n and r) displays concentrated signal at the future aboral end, which is the leading pole during swimming.

There are notable differences between the morphologies of planulae and planuloids. The most obvious difference is that planuloids are much larger than planulae. Planuloids can be over $2 \mathrm{~mm}$ in length and $1 \mathrm{~mm}$ in width at their widest point. Planuloids also contain longitudinal muscle fibers running from the future oral to future aboral end (Figure 9.3p), and no such muscle fibers are present in sexually produced embryos (Figure 9.31). Development of asexual propagules begins with an outpocketing of the body wall of the parent polyp, with the longitudinal muscle fibers of the polyp extending into the developing propagule (Figure 9.30). However, Khabibulina and Starunov (2019) report that these muscle fibers are lost during propagule development, and the fibers observed in the propagule form de novo. Unlike planulae, asexual propagules regularly begin to metamorphose into polyps before attachment to a surface. Finally, asexual propagules may contain symbiotic dinoflagellates in cells of the gastrodermis if the parent polyp is inoculated with symbionts (Figure 9.3p), while planulae only acquire symbionts from the environment once they have developed a mouth in the process of becoming a polyp.

\subsection{ANATOMY}

The C. xamachana body is composed of three layers: epidermis, gastrodermis and mesoglea (Mayer 1910). Planulae are uniformly ciliated and polarized, swimming with the anterior end forward. The anterior end is the precursor to the polyp pedal disk and where settlement occurs. As previously mentioned in this chapter (see Section 9.4), planulae are aposymbiotic and additionally have cnidoblasts (precursors to cnidocytes, the cells which produce cnidocysts or "stinging cells") in their epidermis. Fully differentiated cnidocytes are present in the ectoderm (Martin and Chia 1982). A full description of Cassiopea cnidocysts is located at the end of this section.

After settlement, C. xamachana larvae develop into scyphistomae (polyps). A scyphistoma is composed of a pedal disc securing the polyp to a substrate, a stem rising to meet the head or calyx and a centrally located mouth or hypostome (Figure 9.4a) (Bigelow 1900). The calyx contains four gastric pouches separated by four septal muscles (Bigelow 1892). It has 32 total tentacles: 4 pairs of perradial, 4 pairs of interradial and 8 pairs of adradial tentacles. When fully expanded, the tentacles exceed the length of the body (Bigelow 1900) which is 3 to $4 \mathrm{~mm}$ long with a 1-mmdiameter calyx (head) in fully grown polyps (Figure 9.4a) (Curtis and Cowden 1974). Budding occurs at the base of the calyx in a perradial distribution (Hofmann et al. 1978). The planuloid buds have a single-layered ectoderm with three cell types, an endoderm with two cell types and a thin mesoglea separating the ectoderm from the endoderm. Cnidoblasts are located at the base of the epithelial cells, while cnidocytes are near the epithelial surface (Hofmann and Honegger 1990). While buds detach independently from the polyp, they can form budding chains where two to four buds are connected by ectodermal tubes which eventually sever when the bud detaches. The bud at the base of this chain forms a continuous endoderm with the polyp (Figure 9.4a). Buds are spindle shaped and uniformly ciliated, rotating around a longitudinal axis and swimming with the distal anterior pole forward. This anterior end eventually forms the pedal disc upon settlement (Hofmann et al. 1978).

Symbiosomes localize at the base of a host cell, away from maximum lysosomal activity (Fitt and Trench 1983b). Algae are most dense in the subtentacular region of the polyp and at lowest density in the pedal disk region. The positioning of symbionts ensures transfer of algae to the developing ephyra. Ephyra initially have four simple oral arms with a central mouth opening and develop marginal lobes and rhopalia, the sense-organs of adult C. xamachana (Figure 9.4b-c). 

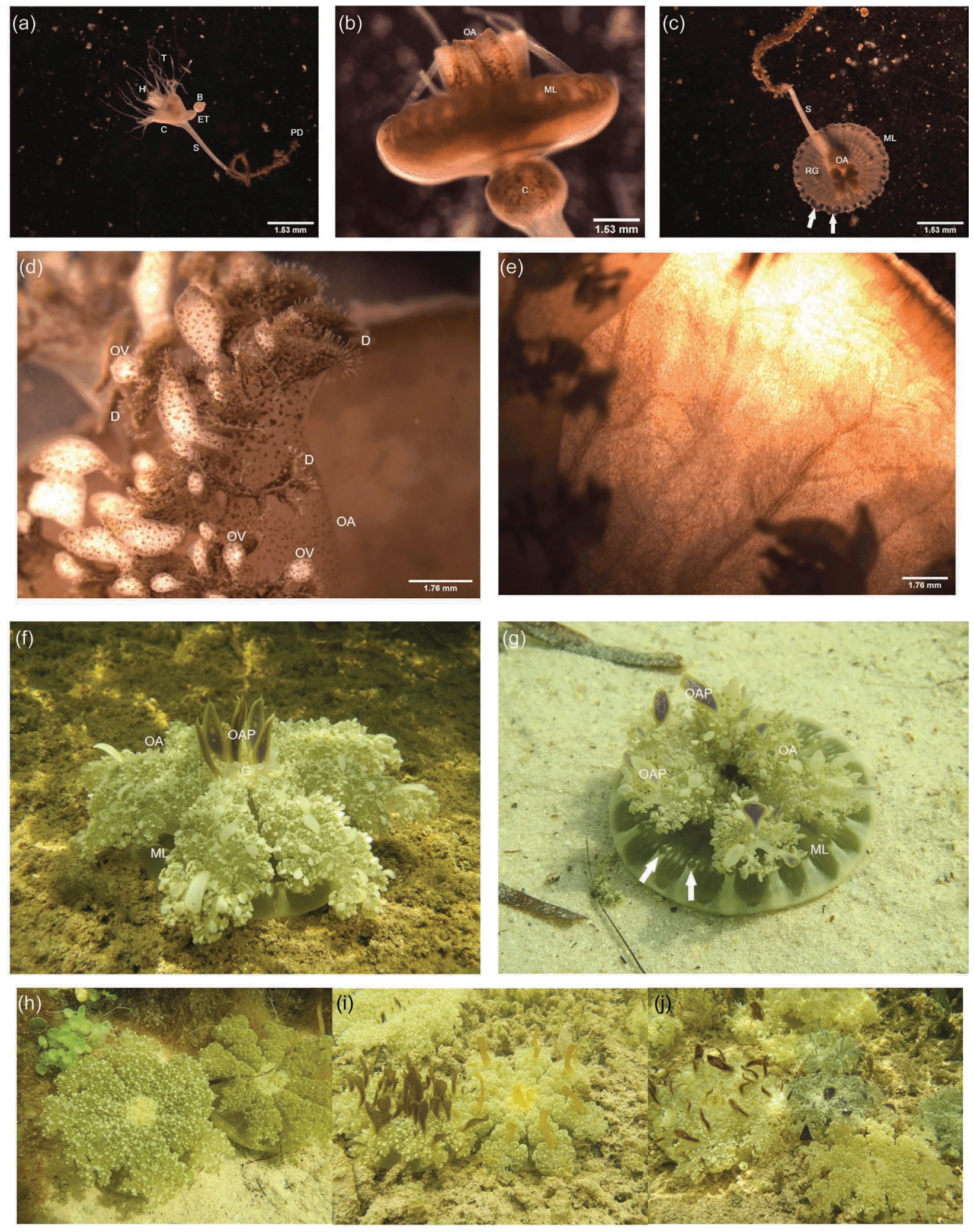

FIGURE 9.4 (a) Aposymbiotic budding scyphistoma. (b) Symbiotic polyp in beginning stages of strobilation. Tentacles have not fully retracted and brown-green algae cells visible within translucent polyp. (c) Symbiotic polyp in late stages of strobilation before ephyra has fully detached. Rhopalia labeled with white arrows. The 32 radial canals are visible on the subumbrella. (d) View of a single oral arm. Symbiont cells are seen within every oral vesicle and the oral arm as a whole. (e) Light passing through the umbrella, highlighting the muscle fibers and also the canal system within. (f-j) Adult Cassiopea photographed in Key Largo, Florida. Multiple color variations and oral appendage distributions seen. Key: H, hypostome; T, tentacles; C, calyx; B, bud; ET, ectodermal tube; S, stem; PD, pedal disc; OA, oral arms; ML, marginal lappets; RG, radial canals; OV, oral vesicles; D, digitata; OAP, oral appendages. 
After detachment of the ephyra, the remaining polyp stem will regenerate a new calyx and tentacles and is capable of strobilating once more, and, in fact, head regeneration has been shown to begin before the strobila fully detaches from the polyp (Hofmann et al. 1978).

While adult $C$. xamachana are physically typical jellyfish, they are unique in that the bell rests on the sandy bottom of their habitats, which has given them the name "upsidedown jellyfish" (Figure 9.4f-j). The adult can secure itself to a surface by using the concave shape of the exumbrella to create suction and adhere to the substrate. The average size of adults seems to vary based on habitat, although a comprehensive size range has not been created to date. Bigelow (1900) reported bell diameter sizes ranging from 6.5 to 24 $\mathrm{cm}$, but Mayer (1910) reports diameters usually around 150 $\mathrm{mm}$. The umbrella perimeter is composed of 80 marginal lappets with corresponding white markings (Figure 9.4g). $C$. xamachana is characterized by its white circular band on the exumbrella, though the exact pattern of these markings differs between individuals. Additionally, there are typically 16 oval-shaped white spots around the umbrella margin corresponding with the rhopalia (sense organs) (Figure 9.4g). Adult $C$. xamachana have on average 16 rhopalia, but individuals have been recorded with anywhere from 10 to 23 rhopalia (Bigelow 1900). Rhopalia are located on notches along the margin of the umbrella and are marked by a reddish-brown pigment spot (Mayer 1910).

Attached to the bell is the oral disc from which the oral arms sprout. Adults have eight oral arms formed in pairs, which are described as rounded and slender compared to those in other Cassiopea species (Figure 9.4f-j). Their length can be greater than the radius of the jellyfish by up to one half. The oral arms have 9 to 15 branches, which are then further branched, giving them a fluffy appearance. Many appendages (oral vesicles) are found at the base of these branches, and they greatly vary in size throughout a jellyfish (Figure 9.4f-j) (Bigelow 1900). The oral arms are also covered with paddle-shaped oral appendages, which are often highly pigmented (Figure $9.4 \mathrm{f}-\mathrm{g}, \mathrm{i}-\mathrm{j}$ ). While $C$. xamachana have reported color morphs of brown and green (Figure $9.4 \mathrm{f}-\mathrm{j}$ ), the morph of deep blue is the most well known and studied. The blue pigment, Cassio Blue, is found in both the oral appendages and diffused within the mesoglea (Blanquet and Phelan 1987). The green and brown morphs have not yet been studied or their pigments characterized, though adult color pattern has been found independent of symbiont species (Lampert et al. 2012).

Brachial canals attach to each pair of arms and converge within the oral disc to empty into the stomach. The stomach contains 32 radial grooves connected by a network of anastomosing branches (Figure 9.4e) (Bigelow 1900). The stomach is surrounded by four subgenital pits and four genital sacs, which are accessible from the outside via four subgenital ducts (Mayer 1910). Adults exhibit sexual dimorphism. Females have visually distinctive brooding appendages, seen as a white cluster of appendages in the center of the oral disc (for more information, see Section 9.4) (Figure 9.4h). The mesoglea makes up most of the body and contains symbiotic cells, which have highest density in the umbrella. An endodermic layer separates the subumbrellar and exumbrellar mesoglea (Bigelow 1900). Muscle fibers cover the subumbrella, and muscle activity has been connected with rhopalia signaling and activity (Mayer 1910). Adults have mostly epitheliomuscular cells with muscle fibers in sheets folded into the mesoglea (Blanquet and Riordan 1981).

Scyphozoan cnidocysts fall into three different categories: isorhizas, anisorhizas and rhopaloids. C. xamachana have three different types of cnidocysts, though the presence and abundance differ based on life stage. Additionally, the names of two of these cnidocysts have been reported differently in literature, and we will list both names for comprehension. Heterotrichous microbasic euryteles (Jensch and Hofmann 1997), or rhopaloids (Ames et al. 2020), are present in the both the ectoderm and endoderm of all life stages. Holotrichous $\alpha$-isorhizas are also found in both the ectoderm and endoderm of the polyp and adult but have not been detected in all parts of the scyphistoma body. Finally, heterotrichous anisorhizas (Jensch and Hofmann 1997), or O-isorhizas (Ames et al. 2020), are only detected in the polyp after strobilation has begun. All three cnidocyst types are found in the adult within the ectoderm, and no cnidocysts are located within the mesoglea of any part of the life cycle. Oral vesicles and adjacent tentacle-like structures called digitata contain clusters of cnidocysts in the ectoderm (Figure 9.4d) (Jensch and Hofmann 1997). These digitata immobilize prey when the natural pulsations of the umbrella pump surrounding water against the oral arms. Additionally, $C$. xamachana ephyrae and adults release large amounts of cnidocyst-containing mucus into the surrounding water upon agitation, a response associated with defense and predation. The undeployed cnidocysts inside this mucus are termed cassiosomes and, unlike the oral arms of the adult, only contain the heterotrichous anisorhiza/Oisorhiza cnidocysts. These cnidocysts line the cassiosome periphery interspaced with ectoderm cells containing cilia, allowing temporary mobility of the unit. The interior space of a cassiosome is mostly empty but uniquely contains symbiont cells. A cassiosome ranges from 100 to $550 \mu \mathrm{m}$ in diameter (Ames et al. 2020). C. xamachana had been reported as both venomous and nonvenomous in different habitats, and potency has been related to venom composition, as the cnidocyst composition is identical between these varieties. C. xamachana stings are described as relatively mild to humans but are capable of hemolytic, proteolytic, cardiotoxic and dermonecrotic effects (Radwan et al. 2001).

\subsection{GENOMIC DATA}

With renewed interest in establishing $C$. xamachana as a model to study cnidarian-dinoflagellate symbiosis, efforts have been put forth to compile genomic and transcriptomic data. The first C. xamachana transcriptomic dataset became publicly available in 2018, and the first Cassiopea genome (T1-A clonal line) was published in 2019 (Kayal et al. 2018; 
Ohdera et al. 2019). The T1-A line is available from the labs of the authors in this chapter. The initial draft genome of $C$. xamachana was composed entirely of Illumina short-read data, resulting in a fragmented assembly $(\mathrm{N} 50=15,563 \mathrm{~Kb})$ compared to the recently published scyphozoan genomes employing third-generation sequencing technology (Gold et al. 2019; Khalturin et al. 2019; Kim et al. 2019; Li et al. 2020). An updated assembly is now available at the US Department of Energy's Joint Genome Institute (JGI)'s web portal, with significant improvements across all assembly statistics $(\mathrm{N} 50=17.8 \mathrm{Mb})$ (https://mycocosm.jgi.doe.gov/ Casxa1). We will continue efforts to improve the assembly and make updates available on the portal. C. xamachana remains the only non-anthozoan cnidarian genome available that establishes a stable symbiosis with Symbiodiniaceae, making it a highly attractive model to study the evolution and genetics of symbiosis. In addition to future resources that will become available, past studies have already begun to utilize and illuminate the genetics underlying Cassiopea.

In silico prediction of the genome size of $C$. xamachana suggests roughly $360 \mathrm{Mb}$, consistent with previous measurements of genome sizes for C. ornata and Cassiopea sp. (Mirsky and Ris 1951; Adachi et al. 2017; Ohdera et al. 2019). A marginally larger assembly of $393.5 \mathrm{Mb}$ was obtained, in line with previous predictions. These values suggest the genus to have genome sizes comparable to other members of the order Rhizostomeae (Kim et al. 2019; Li et al. 2020), but two-fold smaller than the predicted genome size of Aurelia sp1. (Adachi et al. 2017; Gold et al. 2019; Khalturin et al. 2019). A genome size greater than $500 \mathrm{Mb}$ appears to be the exception given the average genome sizes for the two additional Aurelia species sequenced, which may suggest genome size to be relatively constant within the class. Approximately 31,459 protein-coding genes have been predicted from the C. xamachana draft genome, similar to the currently available Aurelia genomes. This is in contrast to its close relatives Nemopilema nomurai and Rhopilema esculentum, which were predicted to contain 18,962 and 17,219 protein coding genes, respectively (Kim et al. 2019; $\mathrm{Li}$ et al. 2020). It remains to be seen whether the ancestor of the suborder Dactyliophorae experienced gene loss or a gene expansion occurred after the split of Kolpophorae.

The gene content and its similarity to bilaterians have prompted researchers to investigate the evolution of genomic organization (Hui et al. 2008; Schierwater and Kuhn 1998; Gauchat et al. 2000; Garcia-Fernàndez 2005). Cnidarians occupy a unique position as sister group to bilaterians. Early investigations into genomic architecture suggested high conservation of protein coding gene between cnidarians and humans despite the large divergence time (Schierwater and Kuhn 1998). A recent analysis of medusozoan genomes showed genetic divergence between major cnidarian lineages to be equivalent to that found in bilaterians (Khalturin et al. 2019). Humans share a remarkable number of genes with jellyfish, offering an opportunity to study the evolution of pre-bilaterian genomic architecture and gene conservation. Ohdera et al. (2018) found nearly 5,000 orthologous gene groups (orthogroups) between cnidarians and humans. C. xamachana in particular shared 444 unique orthogroups with humans, far more than other cnidarian classes. Similar findings were reported for the moon jelly Aurelia aurita, where a high degree of macrosyntenic linkage with humans was found relative to the anemone Nematostella vectensis (Khalturin et al. 2019), suggesting a greater genomic conservation since the cnidarian-bilaterian split. Cnidarians have thus played a crucial role in helping us understand gene family evolution and expansion in metazoans (e.g. Hox genes).

In cnidarians, Hox genes were first recovered from three species of the class Hydrozoa (Schummer et al. 1992), but Cassiopea was the first scyphozoan in which Hox genes were identified (Kuhn et al. 1999). Initial investigations explored how Hox genes may regulate morphological patterning considering the relatively simple body plan. Hox gene expression defines the anterior-posterior axis in Bilateria, and similar regulatory roles have been identified for cnidarian Hox genes (DuBuc et al. 2018; He et al. 2018). As with other cnidarian lineages, Cassiopea maintains a similar repertoire of homeobox genes (Table 9.1). The first homeobox gene identified within Scyphozoa was the Scoxl-5 of Cassiopea (Kuhn et al. 1999), which were grouped within two major cnidarian homeobox groups (Cnoxl, Cnox2). While Cnox2 has since been classified as a parahox gene, all five Cnox groups show highest homology to the bilaterian Antp class of homeobox genes. Moreover, hox gene orientation within clusters is not expressed as such, similar to that seen in bilaterians. In fact, hox expression is not conserved even between cnidarians. It remains to be seen how homeobox genes are involved in strobilation and body polarity. With the improvement in genome quality, investigations of genomic synteny will likely address the questions regarding genomic architecture of the ancestral genome prior to the cnidarian-bilaterian split. Previously, a syntenic linkage between a $P O U$ and Hox gene was thought to have been a pre-bilaterian ancestral feature, as it was found in both vertebrates and the hydrozoan Eleutheria (Kamm and Schierwater 2007). The availability of new medusozoan genomes, including Cassiopea, revealed the linkage may have arisen independently in the medusozoan and vertebrate ancestors (Ohdera et al. 2019).

Another aspect of cnidarian biology that has intrigued biologists is the capacity of Cassiopea to regenerate as well as the lack of senescence. While research has focused largely on Hydra and corals, chromosome specific telomere length was first investigated in Cassiopea (Ojimi and Hidaka 2010). Cassiopea exhibits unequal telomere length depending on life stage, with the bell margin of adult medusae having the longest telomeres (2,000 bp) compared to other tissue types $(\sim 1,200 \mathrm{bp})$. This is despite telomerase activity remaining relatively similar across multiple life-stages (Ojimi et al. 2009). Ojimi et al. (2010) also found the Cassiopea telomeres to resemble the vertebrate sequence (TTAGGG), in agreement with members of other cnidarian classes, suggesting the vertebrate telomere sequence to be ancestral at the cnidarian-bilaterian split (Grant et al. 2003). 
TABLE 9.1

\section{Repertoire of Homeobox Genes in Cnidaria}

\begin{tabular}{|c|c|c|c|c|c|c|c|c|c|c|c|}
\hline & \multirow{2}{*}{\multicolumn{2}{|c|}{$\begin{array}{c}\text { Anthozoa } \\
\begin{array}{l}\text { Exaiptasia Nematostella } \\
\text { diaphana vectensis }\end{array}\end{array}$}} & \multirow{2}{*}{ 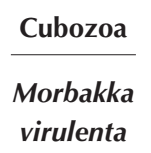 } & \multicolumn{6}{|c|}{ Scyphozoa } & \multicolumn{2}{|c|}{ Hydrozoa } \\
\hline & & & & $\begin{array}{c}\text { Aurelia } \\
\text { sp. } 1\end{array}$ & $\begin{array}{l}\text { Aurelia } \\
\text { aurita }\end{array}$ & $\begin{array}{c}\text { Chrysaora } \\
\text { quinquecirrha }\end{array}$ & $\begin{array}{c}\text { Cassiopea } \\
\text { xamachana }\end{array}$ & $\begin{array}{c}\text { Nemopilema } \\
\text { nomural }\end{array}$ & $\begin{array}{l}\text { Rhopilema } \\
\text { esculentum }\end{array}$ & $\begin{array}{c}\text { Hydra } \\
\text { vulgaris }\end{array}$ & $\begin{array}{c}\text { Clytia } \\
\text { emisphaerica }\end{array}$ \\
\hline ANTP & 62 & 78 & 33 & 35 & 33 & 22 & 32 & 38 & 31 & 17 & 28 \\
\hline CERS & 1 & 1 & 0 & 0 & 0 & 0 & 0 & 0 & 0 & 0 & 0 \\
\hline $\mathrm{HNF}$ & 0 & 1 & 0 & 0 & 0 & 0 & 0 & 0 & 0 & 0 & 0 \\
\hline LIM & 6 & 6 & 5 & 3 & 5 & 3 & 4 & 5 & 5 & 5 & 5 \\
\hline POU & 5 & 6 & 4 & 5 & 3 & 3 & 4 & 3 & 4 & 3 & 4 \\
\hline PRD & 36 & 44 & 25 & 30 & 29 & 22 & 28 & 29 & 20 & 18 & 17 \\
\hline SINE & 4 & 6 & 5 & 4 & 5 & 5 & 4 & 6 & 5 & 2 & 4 \\
\hline TALE & 8 & 5 & 4 & 5 & 7 & 3 & 3 & 7 & 5 & 6 & 5 \\
\hline OTHER & 1 & 4 & 1 & 0 & 0 & 0 & 1 & 0 & 1 & 0 & 0 \\
\hline TOTAL & 123 & 151 & 77 & 82 & 82 & 58 & 76 & 88 & 71 & 51 & 63 \\
\hline
\end{tabular}

Note: Homeobox genes were classified according to the classification outlined by Zhong and Holland (2011), following the method outline by Gold et al. (2019). Protein models from each genome were initially blasted against the curated dataset used by Gold et al. (2019), combined with previously identified cnidarian hox genes from C. xamachana and Aurelia sp1. Matching hits were further assessed using Interpro (https://github.com/ebi-pf-team/interproscan) to confirm the presence of the homeodomain. Genes were further classified using homeoDB (http://homeodb.zoo.ox.ac.uk/) to generate the final counts.

As previously mentioned, species within the order Rhizostomeae are characterized by the blue pigment Cassio Blue. First isolated in Cassiopea and subsequently described in Rhizostoma, Cassio Blue likely plays a photoprotective role (Blanquet and Phelan 1987; Bulina et al. 2004). Researchers also found this chromoprotein to exhibit promiscuous metal binding properties but, strikingly, to contain domains for Frizzled and Kringle, genes involved in wht signaling (Bulina et al. 2004; Phelan et al. 2006). While the function of the chromoprotein beyond its photoprotective role is unknown, the presence of the wnt domains has led to speculation of the protein's additional roles. Given the overlap in protein deposition and symbiont localization, Cassio Blue may be involved in regulation of symbiont density, though this remains to be examined.

The C. xamachana mitochondrial genome was sequenced in 2012 (Kayal et al. 2012). The Cassiopea mitochondrial genome is linear and approximately $17,000 \mathrm{~kb}$ in length (Bridge et al. 1992), with 17 conserved genes and two tRNAs and an intact gene order relative to other medusozoan mitochondrial genomes. Medusozoan mtDNA appears to be streamlined, with short intergenic regions. Scyphozoans including Cassiopea are characterized by a $\sim 90$ bp intergenic region capable of forming a conserved stem loop motif potentially involved in transcriptional regulation and replication. Scyphozoan mtDNAs are also characterized by the presence of a pol-B and ORF314 gene at the chromosome end, a likely signature of an ancient integration of a linear plasmid and consequent linearization of the chromosome. ORF314 may be a terminal protein involved in maintaining mtDNA integrity by binding to the short, inverted terminal repeats at the end of the mtDNA. In addition to gene organization, the COX1 gene has revealed high genetic divergence to exist within the genus. For example, a mean pairwise divergence of $20.3 \%$ was calculated for the two likely invasive species present in Hawaii. This is remarkable considering the morphological similarity between species.

Despite a significant increase in the number of available medusozoan genomes over the past several years. C. xamachana offers a unique position as the sole symbiotic species with a genome currently available. Researchers now have the opportunity to investigate the genetic basis of symbiosis by having access to genomes of different cnidarian lineages exhibiting photosymbiosis with different Symbiodinaceae taxa such as the scyphozoan $C$. xamachana (Ohdera et al. 2019), the sea anemone Exaptasia diaphana (Baumgarten et al. 2015), the octocoral Xenia sp. (Hu et al. 2020) and a growing number of scleractinian corals (e.g. Shinzato et al. 2011; Fuller et al. 2020; Cunning et al. 2018; Shumaker et al. 2018). While the underlying mechanism is yet unclear, the availability of the C. xamachana genome will provide an opportunity to study the convergent evolution of symbiosis within Cnidaria and whether cis- and trans-regulatory mechanisms underlie the evolution of symbiosis within the cnidarian lineage.

\subsection{FUNCTIONAL APPROACHES: TOOLS FOR MOLECULAR AND CELLULAR ANALYSES}

\subsubsection{Toward a Genetic Model to Study CNidarian Symbiosis}

Genetically accessible model organisms have been crucial tools for biologists to understand the molecular underpinnings of life as we know it. Great strides have been made in the past century using genetic model systems to study gene function in other invertebrates, but some systems have not been empowered by these methods. The symbiosis between 
corals and their photosynthetic endosymbionts is the basis of coral reef ecosystems throughout the world, but the absence of genetic tools in a laboratory model system for the investigation of symbiotic cnidarians has prevented a mechanistic understanding of this symbiosis.

Selection of an appropriate laboratory genetic model system is critical for the implementation of genetic tools (Matthews and Vosshall 2020). Successful systems are marked by key features, namely 1) the capacity to close the life cycle in the laboratory, 2) efficient methods for mutagenesis and transgenesis and 3) germline transmission of mutations/transgenes. Reef-building corals generally spawn once annually, with development to sexual maturity requiring multiple years. Infrequent spawning and long generation time impose extreme limitations on hard coral systems for rapid progress in genetics. The anemone Exaiptasia diaphana has been a useful model for cell biology and physiology, but the inability to close the life cycle makes this organism, at present, an intractable system for comprehensive molecular genetic analysis (Jones et al. 2018).

C. xamachana is an apt genetic model system for the study of symbiotic cnidarians. Like reef-building corals, Cassiopea engage in a nutritional endosymbiosis with Symbiodiniaceae and are susceptible to thermal bleaching. However, this organism has multiple characteristics which make it an attractive laboratory system. Cassiopea spawns daily in aquaria (see Section 9.4), providing regular access to single-cell embryos that are necessary to genetically manipulate the organism using microinjection or electroporation (Figure 9.5a-b). The life cycle of this organism has been closed in the laboratory. Development from embryo to polyp (Figure 9.5c-f) and the subsequent formation of ephyrae spans approximately two months. Medusae require additional time to reach sexual maturity, leading to a generation time of fewer than six months. Additionally, polyps can be maintained as immortal lines in the lab, producing buds at rates associated with how much they are fed. Infected scyphistomae can also live forever under constant culture conditions, though in the field, they will be affected by seasonal conditions (e.g. in the Florida Keys, they disappear in the winter months). Medusae require additional time to reach sexual maturity, leading to a generation time of fewer than six months. Given these qualities, Cassiopea provides a practical and relevant model system for a more expedient genetic analysis than in corals. Here we provide some pragmatic information for those interested in using Cassiopea as a laboratory model.

\subsubsection{Establishing a Lab Colony from Wild Collection}

The ability to maintain a breeding $C$. xamachana colonies in relatively simple aquaria is a strength of this model system for cnidarian symbiosis. Reproductive adults can be readily collected from their nearshore natural habitats by snorkeling or wading in the shallow waters they inhabit. In the state of Florida, USA, C. xamachana can be collected under a recreational saltwater fishing license. For the purposes of lab-based spawning, medusae from $10-15 \mathrm{~cm}$ in bell diameter are appropriate for long-term culture in aquaria. Males and females can be readily identified via externally visible morphological characteristics, namely the presence of central brooding appendages on females (Hofmann and Hadfield 2002). While larger individuals can be kept, their higher biomass and food requirements make them less conducive to sustained culture in closed systems. Medusae can be shipped overnight and fare well when packaged inside of individual poly bags, approximately half filled with water to allow for airspace for gas exchange, shipped inside of an insulated foam box to stabilize temperature during the journey.

\subsubsection{Culturing Cassiopea in the Lab}

A stable, purpose-built aquarium system greatly facilitates the maintenance of a spawning C. xamachana colony. Overall, these organisms fare well with high levels of light (250-400 $\mu \mathrm{E} \mathrm{m}^{-2} \mathrm{~s}^{-1}$ ), frequent and heavy feeding (freshly hatched Artemia sp. Nauplii, which can be supplemented with rotifers) and low water flow. A shallow tank with a plumbed sump functions well as a foundation for a colony, with a few considerations of our organism. While relatively robust, C. xamachana will readily be pulled into overflows as well as powerheads and other circulation pumps. Long, shallow tanks of $15-30 \mathrm{~cm}$ depth provide convenient access and reduce crowding. No powerheads, pumps or other equipment should be located directly in the tank. The overflow which brings water from the tank to the sump via gravity should be covered with a protective grate constructed from polystyrene egg crate lighting diffuser. In the sump, water first passes through a filter sock or floss, which should be washed/exchanged at least every other day. The sump also contains live rock or other media to serve as biological filtration, as well as an efficient and appropriately sized protein skimmer which both removes waste and facilitates gas exchange. A temperature of $25-26^{\circ} \mathrm{C}$ is maintained with an aquarium heater located in the sump. As aquarium heaters are notoriously unreliable and failure in the on position may result in severe impacts to the colony, the heater should be backed up by a secondary temperature controller. Activated carbon is also located in the sump in order to remove organics that reduce water clarity; this should be kept in a filter bag or nylons and changed monthly; approximately $60 \mathrm{~mL}$ per 100 liters of water in the system is sufficient. The return pump delivers water back to the aquarium. This should be relatively low flow so as not to unnecessarily disturb the medusae in the main tank; approximate turnover of one to three times the volume of the aquarium is sufficient. Diffusing the water returning to the tank will also prevent the disturbance of the medusae (Widmer 2008).

Heavy feeding of freshly hatched live Artemia sp. one to three times daily facilitates continued, regular spawning. Though C. xamachana are not particularly demanding of water quality, attention to water parameters will promote the 

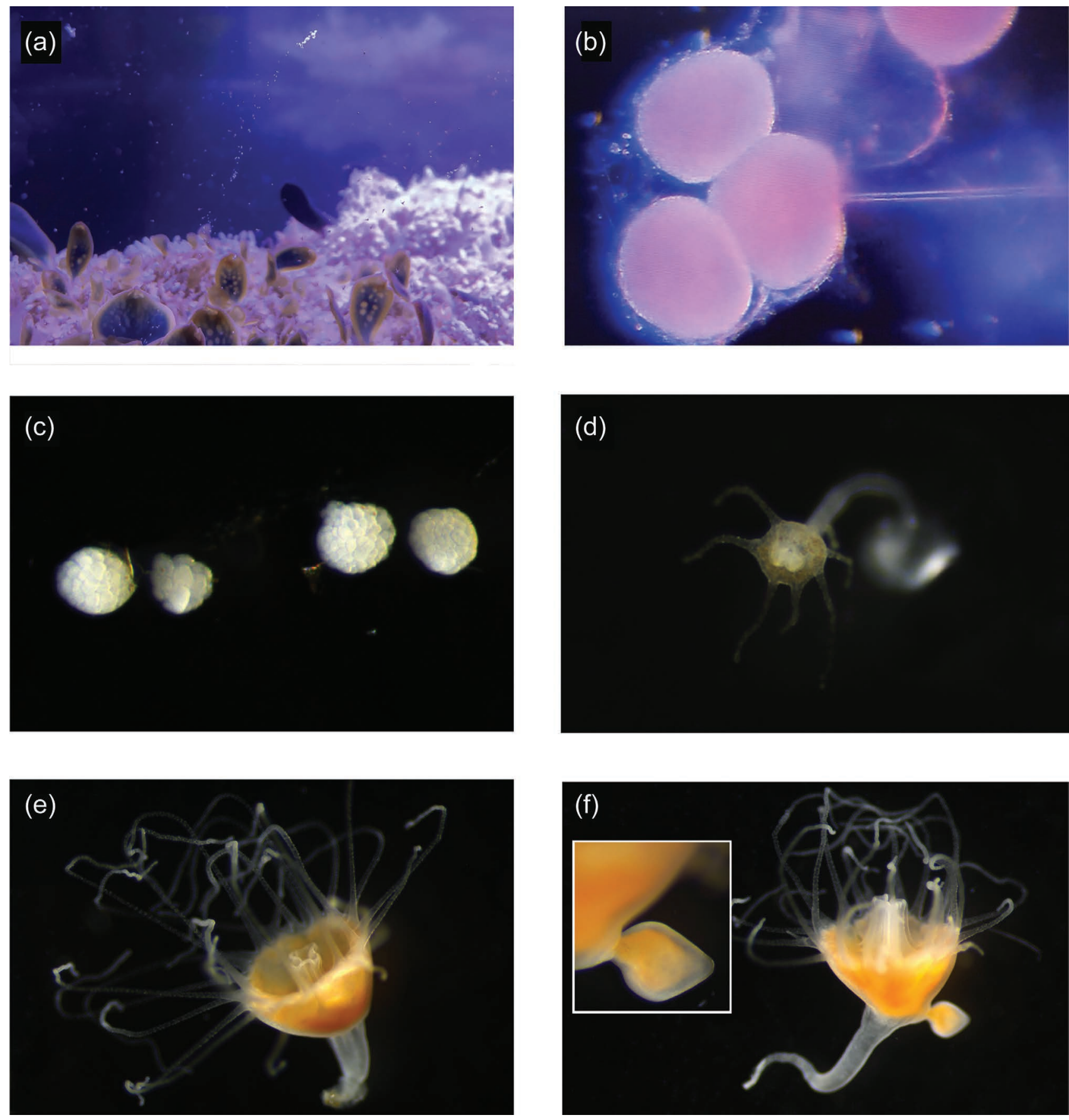

FIGURE 9.5 Spawning, injection and settlement of Cassiopea. (a) Daily spawning of Cassiopea in the laboratory environment. (b) Injection of Cas9-RNPs into single-cell embryos, with visualization aided by phenol red tracer dye. (c) Development of injected embryos, ten hours after injection. (d) Metamorphosis and settlement of injected Cassiopea embryo into a small polyp, ten days following injection. (e) Growth of an injected embryo into a polyp, 30 days after injection. (f) Development of asexual planuloid buds on a polyp (see inset for detail) 45 days following injection.

longevity of the culture and consistent spawning. Artificial seawater should be mixed using 0 TDS RO/DI water to a salinity of 34-36 PSU. Weekly water changes of $20 \%$ are helpful in long-term maintenance and stability. Nitrate and phosphate levels should be monitored weekly; low or high levels can be problematic. As a guideline, nitrate levels of 2-10 ppm and phosphate levels of 0.03 to $0.10 \mathrm{ppm}$ have provided for consistent maintenance and spawning of broodstock. Excess nutrients can be managed by increasing the volume of water changes and implementing an algal refugium (e.g. Chaetomorpha) in the sump. Insufficient nutrients in the water can be ameliorated by increasing feeding, reducing skimming or with the careful dosing of sodium nitrate or sodium phosphate solutions to achieve desired levels. 
As photosymbiotic organisms with spawning controlled by the daily light cycle, appropriate lighting is a critical component of Cassiopea husbandry. Lighting solutions designed for reef-building corals are appropriate for these shallowwater animals that require high levels of photosynthetically available radiation (PAR) to maximize the nutritional benefits from their endosymbionts. Modern high-output LEDs designed for reef tanks can be implemented to blanket the bottom of the tank with PAR levels of $250-400 \mu \mathrm{E}$ on a 12:12 daily cycle. Light levels should be assessed with a submersible PAR meter and lighting adjusted as appropriate.

\subsubsection{Microinjection of Single-Cell Embryos for the Generations of Mutants and Transgenic Cassiopea}

The study of symbiosis in cnidarians has long sought to identify the mechanistic basis of the interactions between the animal host and intracellular algal partner. Studies comparing symbiotic and aposymbiotic hosts have been performed in numerous cnidarian taxa (Lehnert et al. 2014; RodriguezLanetty et al. 2006), as well as numerous studies examining the response to heat stress and the breakdown of symbiosis (Pinzón et al. 2015; DeSalvo et al. 2010) and gene expression patterns associated with thermal tolerance (Bellantuono et al. 2012; Barshis et al. 2013). This broad body of work has resulted in the identification of numerous genes of interest, including molecular chaperones and antioxidant enzymes associated with the response to thermal stress (Császár et al. 2009; Fang et al. 1997), as well as lectins which may mediate the relationship between the host and symbiont (Kvennefors et al. 2008). However, the field has largely been missing crucial tools of genetics to robustly test these hypotheses. Microinjection of C. xamachana embryos opens a path to understand the molecular genetic basis of symbiosis, chemosensation and sleep in an early diverging metazoan with a decentralized nervous system (Figure 9.5b).

A basic tool of genetics is the capacity to perform lossof-function studies such as gene knockout experiments. With the development of genome editing techniques, $C$. xamachana is an apt model system to test hypotheses of cnidarian symbiosis. Using microinjection, C. xamachana embryos are amenable to CRISPR-mediated mutagenesis, a technology which allows for precise, targeted mutagenesis and transgenesis using a programmable nuclease comprised of a guide RNA and the protein Cas9 (Jinek et al. 2012). CRISPR-Cas 9 can be used by delivering the Cas 9 protein complexed with single guide RNAs (sgRNA) which direct the nuclease to the locus of interest in the nucleus of a living cell. This Cas9-sgRNA complex cleaves the targeted DNA, resulting in endogenous DNA repair. In the absence of homologous template, non-homologous end joining (NHEJ) repair occurs (Doudna and Charpentier 2014). By injecting a Cas9-sgRNA complex into single cell embryos, mutants are generated with small insertions or deletions (indels) induced by the imperfect DNA repair mechanisms of the cell. These indels often result in frameshift mutations of the target gene, generating loss-of-function alleles. These mutagenized embryos can then be reared to polyps and induced to strobilate by exposure to an algal symbiont, generating medusae that can be used for subsequent crosses once sexually mature. As the life cycle of Cassiopea can be completed in the lab within four to six months, the crosses necessary to generate a homozygous mutant can be completed within 18 months. Work to establish this technology in Cassiopea is ongoing.

In addition to using CRISPR to generate loss-of-function alleles, this technology can also be implemented to perform gene knock-in. By providing donor DNA consisting of a transgene flanked by sequence homologous to the both sides of the cut site, CRISPR can be used to engineer knock-in at a specific locus (Barrangou and Doudna 2016). This will allow the generation of diverse molecular tools for Cassiopea for the study of cnidarian symbiosis, development and neuroscience in this unique model system with the future implementation of genetically encoded calcium indicators (GECIs) such as GCaMP (Nakai et al. 2001) for the real-time fluorescent readout of nervous system activity, as well as genetically encoded fluorescent redox sensors (Lukyanov and Belousov 2014) to test longstanding hypotheses regarding the role of ROS stress in cnidarian bleaching. Cassiopea are transparent and lack endogenous host autofluorescence, making them well suited to molecular imaging.

Spawning is timed by the daily light cycle, occurring five to six hours after artificial sunrise in aquaria. In order to collect unicellular embryos, clear selected spawning female medusae of previously extruded, multicellular embryos approximately two hours prior to spawning using a baster. Selected female medusae can then be placed in shallow black polycarbonate pans under a light source to improve the visibility of embryos at the time of release. Once released, the $80-\mu \mathrm{m}$ embryos can be collected with a transfer pipette into small glass dishes, taking care to avoid mucus. Prior to injection, unicellular embryos are transferred and aligned in polystyrene culture dishes containing 40 PSU seawater. The increased salinity results in a slight reduction of cell volume due to osmosis and allows the cell to accommodate the volume of the injected liquid payload. Transfer and positioning of embryos is performed using an aspirator constructed from a 1-mm glass capillary fitted with a length of 1-mm ID silicone tubing. Embryos readily adhere to new, virgin polystyrene and can be arranged in a row for efficient microinjection. Dishes with tight-fitting lids are best employed to reduce evaporation, as the injection dish also houses embryos during development to planulae.

Typical injection payloads include Cas9-sgRNA ribonucleoprotein injection mixture, composed of a guide RNA complexed with Cas9 protein (with NLS), injection buffer and phenol red dye microinjected into single-cell Cassiopea embryos (Figure 9.5b-c). Custom needles are prepared with thin-walled 1-mm aluminosilicate glass capillaries on a P-1000 horizontal pipette puller (Sutter Instrument, CA, USA) and beveled on BV-10 micropipette beveler (Sutter) to $17^{\circ}$. Microinjection is performed using a Xenoworks digital 
injector and manipulator system (Sutter Instrument, CA, USA) under a SteREO Discovery V8 microscope (Zeiss, Germany). Current injection methods yield survival rates of up to $40 \%$. In the three to six hours following injection, each embryo is examined to assess whether it has survived and entered the cleavage stage. Non-dividing embryos are culled and removed, and the water in the dish is carefully replaced with filtered 34 PSU artificial seawater. Planulation of viable embryos occurs approximately one week following injection, with a developmental delay often observed in comparison to uninjected embryos. Cassiopea larvae readily settle and metamorphose in response to a number of cues, including bacteria, degrading mangrove leaves and the previously mentioned endogenous metamorphosis-inducing peptide (Neumann 1979; Fleck and Fitt 1999; Thieme and Hofmann 2003). We have found that settlement dishes can easily be prepared by using a cotton swab to transfer biofilm from the sump of an established Cassiopea tank to polystyrene dishes and then covering with seawater and incubating at room temperature for three to five days. Prior to transferring planulae to settlement dishes, water should be exchanged with filtered 34 PSU artificial seawater. Planulae should be monitored regularly; once settlement occurs and nascent scyphistomae have developed tentacles, regular feeding of freshly hatched Artemia nauplii should begin. Daily feeding is optimal. The survival of recent settlers can be enhanced by placing a nauplius on the hypostome with forceps. In order to maintain polyps in an aposymbiotic state and prevent strobilation, polyps can be maintained in $10 \mu \mathrm{m}$ DCMU without apparent detriment. In order to generate medusae, mature polyps can be challenged with symbionts to induce strobilation. Once released from the polyp, the ephyra will develop into a medusa. Growth is facilitated with ample feeding of Artemia (at least daily) and high artificial light levels $(250-400 \mu \mathrm{E})$ on a 12:12 cycle or natural light. With regular water changes, medusae can be cultured in 1-liter beakers or polycarbonate pans to bell diameters of at least $5 \mathrm{~cm}$. The generation of sexually mature medusae takes several months. Work is in progress to develop the most efficient methods to cross medusae.

\subsection{CHALLENGING QUESTIONS}

While a lot of emphasis has been placed on understanding the origins of the first metazoan body plans, less is known about how those early animals interacted with their surrounding microbial seas. The establishment of holobiont communities (i.e. a multicellular host and its associated microbiome) required the evolution of novel interkingdom communication. As metazoan life cycles evolved, their associated microbial communities diversified with them (McFall-Ngai et al. 2013). The study of host-microbe associations throughout an organism's life cycle is now feasible (Gilbert et al. 2015; Gilbert 2016). There is a growing interest in ontogenetic microbiomes (i.e. microbial associates over a host developmental time course) (Fieth et al. 2016; Carrier and Reitzel 2018; Vijayan et al. 2019) and how they can affect developmental phenotypes (Tran and Hadfield 2011; Thompson et al. 2015; Fieth et al. 2016; Shikuma et al. 2016; Carrier and Reitzel 2018). While a few microbes have been shown to induce larval settlement in C. xamachana, such as Vibrio spp. (Neumann 1979; Hofmann and Brand 1987) and Pseudoalteromonas sp. (Ohdera et al. in prep a), it is likely that the complex microbiomes in settlement substrates as well as developmental microbiomes acquired by the organism through ontogeny will also play critical roles in driving phenotypic and physiological traits as C. xamachana goes through its life cycle (Medina lab, unpublished). Our ability to infect with different Symbiodiniaceae that will in turn harbor different microbiomes as well as potentially developing axenic and gnotobiotic animals will also open doors to understand host-microbiome interactions at the developmental level (Medina lab, unpublished).

Many cnidarian taxa establish endosymbioses with Symbiodiniaceae, and this symbiosis is crucial in the maintenance of coral reef ecosystems (LaJeunesse 2020). Scleractinian corals usually establish their photosymbiosis during the larval stage (Schwarz et al. 1999; Abrego et al. 2009; Voolstra et al. 2009; McIlroy and Coffroth 2017). Mounting evidence now supports the role of Symbiodiniaceae (LaJeunesse et al. 2018) in the onset of host development (Mohamed et al. 2016; Reich et al. 2017). Coral larval manipulation experiments are challenging given the limited availability of larvae due to annual spawning events (Harrison et al. 1984; Szmant 1986; Van Woesik et al. 2006). Although the pelago-benthic transition from larva to settled polyp is partially linked to onset of photosymbiosis (Mohamed et al. 2016; Reich et al. 2017), discerning the role of photosymbionts as drivers of this developmental transition has not been clearly elucidated (Hartmann et al. 2019). Cassiopea therefore represents an efficient model system to study developmental symbioses.

We believe that $C$. xamachana can become an ideal system to study environmental canalization (Waddington 1942) because of the clear and easily manipulated developmental switch (i.e. onset of photosymbiosis) that we can also obviate with artificial inducers. We can alter the phenotypic outcome of strobilation by using different photosymbionts in comparative infection experiments. Once the polyp stage is infected, it can take different developmental trajectories that lead to divergent morphospaces between homologous and heterologous photosymbiotic infections (Figure 9.6). These different developmental phenotypes also likely have diverging underlying molecular regulatory mechanisms. Robert Trench had indeed already proposed that this type of photosymbiosis would be ideal for the study of cross-genome regulation (Trench 1979). In support of this idea, we have uncovered a possible role of $S$. microadriaticum photosynthetic pigments in the regulation of $C$. xamachana strobilation (Ohdera et al. in prep b).

Both the host (C. xamachana) (Ohdera et al. 2019) and the homologous photosymbiont (S. microadriaticum) (Aranda et al. 2016) are now genome enabled, facilitating any downstream molecular analysis. Establishing 


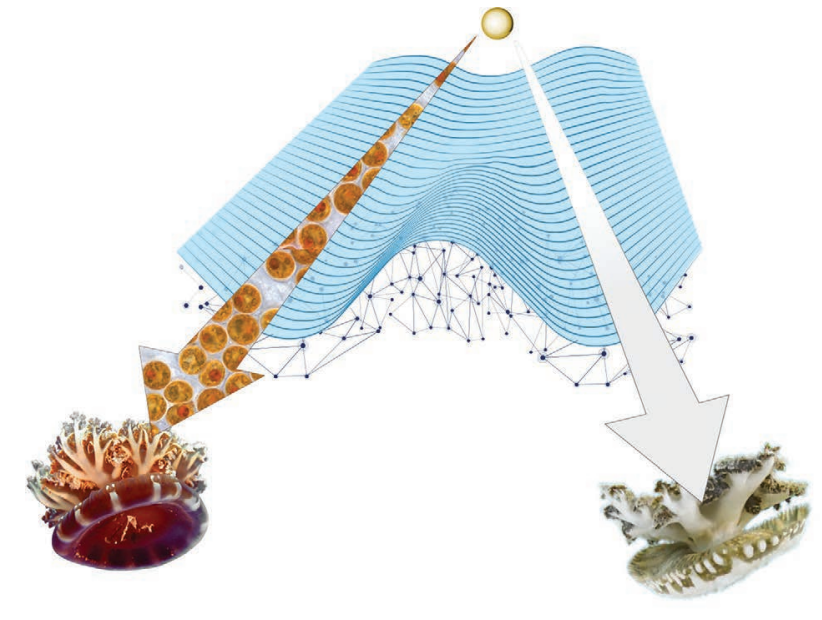

FIGURE 9.6 Symbiosis-driven development in C. xamachana. The small white circle represents the zygote stage that follows different developmental trajectories. Strobilation can lead to different phenotypic outcomes (i.e. symbiotic vs. aposymbiotic strobila) driven by photosymbiosis vs environmental and/or chemical cues. The symbiotic route is the one that occurs primarily in nature. The aposymbiotic route can be lab induced and is probably environmentally induced as well. The underlying genetic network is therefore dynamic and slightly modified depending on the trigger of strobilation.

laboratory lines of both host and photosymbionts has been straightforward, and we can complete the C. xamachana life cycle in the lab in which aposymbiotic asexual polyps (scyphistomae) metamorphose (strobilation) into sexual medusae (ephyrae) due to onset of photosymbiosis (Figure 9.2). Cell-type specific genes have not yet been identified in C. xamachana; however, single-cell transcriptomics has already been successfully used for the study of other cnidarian symbiosis (Hu et al. 2020) and can therefore readily be implemented in the upside-down jellyfish. We can now also chemically induce strobilation (Cabrales-Arellano et al. 2017), providing a suitable control for the study of photosymbiosis-driven development. In addition to onset of developmental symbiosis, we are able to perform timely thermal stress (disruption of symbiosis) experiments that can shed light on the mechanism of cnidarian bleaching affecting coral reefs worldwide due to climate change (Newkirk et al. 2020).

The nervous system is a key driver of animal responses to environmental changes; Cassiopea and other cnidarians are likely to be no exception. The roles of circadian rhythm and sleep in a photosymbiotic animal have only begun to be characterized. C. xamachana is the earliest branching metazoan to exhibit sleep (Nath et al. 2017) that coincidentally is also symbiotic. Thus, of particular interest is host cellular responses to photosynthetic products from the algal symbiont (Ohdera et al. in prep b). In addition, the sensory biology of cnidarians is poorly understood. How the animal may sense heat or chemical stressors may have an impact on the maintenance of symbiosis.
Regeneration has been reported in C. xamachana since the turn of the 20th century (Mayer 1908; Stockard 1910; Cary 1916; Curtis and Cowden 1974; Gamero et al. 2019), but the environmental and molecular drivers of regeneration have not been tackled in this organism. Thus, it is not well known how regeneration progresses and how to successfully induce it in lab. It is still unknown whether $C$. xamachana has stem cells and, if so, what type and where they are generated. Metazoan regeneration (Li et al. 2015; Tiozzo and Copley 2015) is a burgeoning field thanks to increasingly readily available genomic tools for diverse taxa (e.g. Shao et al. 2020; Medina-Feliciano et al. 2020; Gerhke et al. 2019) and increased awareness of the importance of new relevant model systems (Sanchez-Alvarado 2004). Studies of regeneration in C. xamachana can provide a new perspective by being a symbiotic organism as well as basal animal that can shed light in possible shared regenerative traits in the prebilaterian ancestor.

As mentioned earlier in the chapter, $C$. xamachana sexual reproduction in the field and lab still needs additional research. We have yet to uncover when and what triggers male sperm release in the wild. Fertilization is internal, and it is unknown what the female attractants are and when exactly it takes place. Uncovering these aspects of sexual reproduction will yield knowledge useful in understanding gamete recognition in marine taxa, possibly understanding if hybrids can form between congeneric species and improving husbandry techniques.

Adult C. xamachana phenotypic plasticity in color morphotypes and variation in number and size of lappets (Figure 9.4f-j) becomes more apparent at densely populated sites. The vast variation of color morphotypes deserves investigation to understand whether coloration is inherited or environmentally driven and how much of this variation is linked to the photosymbiosis life style. These chromoproteins can potentially have biotechnological application.

In summary, there are many aspects of cnidarian and photosymbiosis biology that will be better understood with the use of $C$. xamachana as a model system. The growing Cassiopea scientific community holds an annual workshop at the Key Largo Marine Research Lab every year where participants can exchange ideas and perform experiments on the readily available Cassiopea population. Additional information about the workshop and resources can be found at http://cassiopeabase.org/. We hope this chapter offers enough information for the community to implement the use of C. xamachana as a model system in labs around the world.

\section{ACKNOWLEDGMENTS}

We thank Justin Wheeler for help with the design of Figure 9.6. Igor Grigoriev and Sajeet Haridas released the JGI $C$. xamachana genome assembly in time for the publication of this chapter. M. Medina was funded by NSF grants OCE 1442206 and OCE 1642311. A.C. Morandini was funded 
by CNPq 309440/2019-0 and FAPESP 2011/50242-5, 2015/21007-9. We thank Key Largo Marine Research Lab for the incessant hospitality over the years. D. Hoffman also extends his thanks and deep appreciation to Dr. Bill Fitt for decades of joint research on Cassiopea.

\section{BIBLIOGRAPHY}

Abrego, D., M. J. H. Van Oppen and B. L. Willis. 2009. Onset of algal endosymbiont specificity varies among closely related species of Acropora corals during early ontogeny. Molecular Ecology 18: 3532-3543.

Adachi, K., H. Miyake, T. Kuramochi, et al. 2017. Genome size distribution in phylum Cnidaria. Fisheries Science 83: 107-112.

Agassiz, A. and A. G. Mayer. 1899. Acalephs from the Fiji Islands. 33: $157-189$.

Al-jbour, S. M., M. Zimmer and A. Kunzmann. 2017. Cellular respiration, oxygen consumption, and trade-offs of the jellyfish Cassiopea sp. in response to temperature change. Journal of Sea Research 128: 92-97.

Ames, C. L., A. M. L. Klompen, K. Badhiwala, et al. 2020. Cassiosomes are stinging-cell structures in the mucus of the upside-down jellyfish Cassiopea xamachana. Communications Biology 3: 67.

Arai, M. N. 2001. Pelagic coelenterates and eutrophication: A review. In: Hydrobiologia 451: 69-87.

Arai, Y., R. Gotoh, J. Yokoyama, et al. 2017. Phylogenetic relationships and morphological variations of upside-down jellyfishes, Cassiopea spp. inhabiting Palau Islands. Biogeography 10: 133-141.

Aranda, M., Y. Li, Y. J. Liew, et al. 2016. Genomes of coral dinoflagellate symbionts highlight evolutionary adaptations conducive to a symbiotic lifestyle. Scientific Reports 6: 39734.

Baker, H. G. 1955. Self-compatibility and establishment after "long-distance" dispersal. Evolution 9: 347-349.

Baker, H. G. 1965. Characteristics and modes of origin of weeds, In The genetics of colonizing species.

Balderston, L. and G. Claus 1969. Study of symbiotic relationship between Symbiodinium microadriaticum Freudenthal, zooxanthella and upside down jellyfish. Nova Hedwigia 17: 373-382.

Barrangou, R. and J. A. Doudna. 2016. Applications of CRISPR technologies in research and beyond. Nature Biotechnology 34: 933-941.

Barshis, D. J., J. T. Ladner, T. A. Oliver, et al. 2013. Genomic basis for coral resilience to climate change. Proceedings of the National Academy of Sciences of the United States of America 110: 1387-1392.

Baumgarten, S., O. Simakov, L. Y. Esherick, et al. 2015. The genome of Aiptasia, a sea anemone model for coral symbiosis. Proceedings of the National Academy of Sciences 112: 11893-11898.

Bayha, K. M. and W. M. Graham. 2014. Nonindigenous marine jellyfish: invasiveness, invasibility, and impacts. In Jellyfish blooms, K. A. Pitt and C. H. Lucas (Eds.), pp. 45-78. Springer.

Bellantuono, A. J., C. Granados-Cifuentes, D. J. Miller, et al. 2012. Coral thermal tolerance: Tuning gene expression to resist thermal stress. PLoS One 7, Nr. 11.

Bigelow, R. P. 1892. On a new species of Cassiopea from Jamaica. Zoologischer Anzeiger 15: 212-214.

Bigelow, R. P. 1893. Some observations on Polyclonia frondosa. Baltimore: John Hopkins University.

Bigelow, R. P. 1900. The anatomy and development of Cassiopea xamachana. Boston Society of Natural History.
Blanquet, R. S. and M. A. Phelan. 1987. An unusual blue mesogleal protein from the mangrove jellyfish Cassiopea xamachana. Marine Biology 94: 423-430.

Blanquet, R. S. and G. P. Riordan. 1981. An ultrastructural study of the subumbrellar musculature and desmosomal complexes of Cassiopea xamachana (Cnidaria: Scyphozoa). Transactions of the American Microscopical Society 100: 109-119.

Brandt, J. F. 1835. Prodromus Descriptionis Animalium Ab H. Mertensio in Orbis Terrarum Circumnavigatione Observatorum.

Brandt, J. F. and C. H. Mertens. 1838. Ausführliche Beschreibung der von $\mathrm{CH}$ Mertens auf seiner Weltumsegelung beobachteten Schirmquallen: Nebst allgemeinen Bemerkungen über die Schirmquallen überhaupt. 2: 237-411.

Bridge, D., C. W. Cunningham, B. Schierwater et al. 1992. Classlevel relationships in the phylum Cnidaria: Evidence from mitochondrial genome structure. Proceedings of the National Academy of Sciences of the United States of America 89: 8750-8753.

Bulina, M. E., K. A. Lukyanov, I. V. Yampolsky, et al. 2004. New class of blue animal pigments based on Frizzled and Kringle protein domains. Journal of Biological Chemistry 279: 43367-43370.

Cabrales-Arellano, P., T. Islas-Flores, P. E. Thomé, et al. 2017. Indomethacin reproducibly induces metamorphosis in Cassiopea xamachana scyphistomae. PeerJ, Nr. 3.

Carrier, T. J. and A. M. Reitzel. 2018. Convergent shifts in hostassociated microbial communities across environmentally elicited phenotypes. Nature Communications 9: 952.

Cary, L. R. 1916. The influence of the marginal sense organs on the rate of regeneration in Cassiopea xamachana. Journal of Experimental Zoology 21: 1-32.

Cary, L. R. 1917. Studies on the physiology of the nervous system of Cassiopea xamachana. Carnegie Institution of Washington Plub, Nr 251: 121-170.

Çevik, C., I. T. Erkol and B. Toklu. 2006. A new record of an alien jellyfish from the Levantine coast of Turkey-Cassiopea andromeda (Forskål, 1775) [Cnidaria: Scyphozoa: Rhizostomea]. Aquatic Invasions 1, Nr. 3.

Colley, N. J. and R. K. Trench. 1983. Selectivity in phagocytosis and persistence of symbiotic algae in the scyphistoma stage of the jellyfish Cassiopeia xamachana. Proceedings of the Royal Society of London. Series B, Containing papers of a Biological character. Royal Society (Great Britain) 219: 61-82.

Colley, N. J. and R. K. Trench. 1985. Cellular events in the reestablishment of a symbiosis between a marine dinoflagellate and a coelenterate. Cell and Tissue Research 239: 93-103.

Császár, N. B. M., F. O. Seneca and M. J. H. Van Oppen. 2009. Variation in antioxidant gene expression in the scleractinian coral Acropora millepora under laboratory thermal stress. Marine Ecology Progress Series 392: 93-102.

Cunning, R., R. A. Bay, P. Gillette, et al. 2018. Comparative analysis of the Pocillopora damicornis genome highlights role of immune system in coral evolution. Scientific Reports 8: 16134.

Curtis, S. K. and R. R. Cowden. 1971. Normal and experimentally modified development of buds in Cassiopea (phylum Coelenterata; class Scyphozoa). Acta embryologiae experimentalis 3: 239-259.

Curtis, S. K. and R. R. Cowden. 1972. Regenerative capacities of the scyphistoma of Cassiopea (Phylum, Coelenterata; class, Scyphozoa). Acta embryologiae experimentalis 1972: 429-454.

Curtis, S. K. and R. R. Cowden. 1974. Some aspects of regeneration in the scyphistoma of Cassiopea (class Scyphozoa) as 
revealed by the use of antimetabolites and microspectrophotometry. American Zoologist 14: 851-866.

DeSalvo, M. K., S. Sunagawa, P. L. Fisher, et al. 2010. Coral host transcriptomic states are correlated with Symbiodinium genotypes. Molecular Ecology 19: 1174-1186.

Doty, M. S. 1961. Acanthophora, a possible invader of the marine flora of Hawaii. Pacific Science 15: 547-552.

Doudna, J. A. and E. Charpentier. 2014. The new frontier of genome engineering with CRISPR-Cas9. Science 346: 1258096.

Dubuc, T. Q., T. B. Stephenson, A. Q. Rock, et al. 2018. Hox and Wnt pattern the primary body axis of an anthozoan cnidarian before gastrulation. Nature Communications 9, Nr. 1 .

Fang, L. S., S. P. Huang and K. L. Lin. 1997. High temperature induces the synthesis of heat-shock proteins and the elevation of intracellular calcium in the coral Acropora grandis. Coral Reefs 16: 127-131.

Fieth, R. A., M. E. A. Gauthier, J. Bayes, et al. 2016. Ontogenetic changes in the bacterial symbiont community of the tropical demosponge Amphimedon queenslandica: Metamorphosis is a new beginning. Frontiers in Marine Science 3: 228.

Fitt, W. K. 1984. The role of chemosensory behavior of Symbiodinium microadriaticum, intermediate hosts, and host behavior in the infection of coelenterates and molluscs with zooxanthellae. Marine Biology 81: 9-17.

Fitt, W. K. 1985. Effect of different strains of the zooxanthellae Symbiodinium microadriaticum on growth and survival of their coelenterate and molluscan hosts. Proceedings of the 5th International Coral Reef Congress, 6: 228.

Fitt, W. K. and R. K. Trench. 1983a. Endocytosis of the symbiotic dinoflagellate Symbiodinium microadriaticum Freudenthal by endodermal cells of the scyphistomae of Cassiopeia xamachana and resistance of the algae to host digestion. Journal of Cell Science 64: 195-212.

Fitt, W. K. and R. K. Trench. 1983b. Infection of coelenterate hosts with the symbiotic dinoflagellate Symbiodinium microadriaticum. In: Intracellular space as oligogenetic ecosystem. Proceedings, 2: 675-681.

Fitt, W. K. and K. Costley. 1998. The role of temperature in survival of the polyp stage of the tropical rhizostome jellyfish Cassiopea xamachana. Journal of Experimental Marine Biology and Ecology 222: 79-91.

Fitt, W. K. and D. K. Hofmann. 1985. Chemical induction of settlement and metamorphosis of planulae and buds of the reefdwelling coelenterate Cassiopeia andromeda. Proceedings of the 5th International Coral Reef Congress, 5: 239-244, Tahiti.

Fitt, W. K., D. K. Hofmann and M. Rahat. 1987. Requirement of exogenous inducers for metamorphosis of axenic larvae and buds of Cassiopeia andromeda (Cnidaria: Scyphozoan). Marine Biology 94: 415-422.

Fleck, J. and A. Bischoff. 1993. Protein kinase C is possibly involved in chemical induction of metamorphosis in Cassiopea spp. (Cnidaria: Scyphozoa). Proceedings of the 5th International Coral Reef Congress, 456-462. Richmond RH (ed) University of Guam Press, KOG Station, Mangilao, Guam, USA.

Fleck, J., W. K. Fitt and M. G. Hahn. 1999. A proline-rich peptide originating from decomposing mangrove leaves is one natural metamorphic cue of the tropical jellyfish Cassiopea xamachana. Marine Ecology Progress Series 183: 115-124.

Fleck, J. and W. K. Fitt. 1999. Degrading mangrove leaves of Rhizophora mangle Linne provide a natural cue for settlement and metamorphosis of the upside down jellyfish Cassiopea xamachana. Journal of Experimental Marine Biology and Ecology 234: 83-94.

Fleck, J. and D. K. Hofmann. 1995. In vivo binding of a biologically active oligopeptide in vegetative buds of the scyphozoan Cassiopea andromeda: Demonstration of receptor-mediated induction of metamorphosis. Marine Biology 122: 447-451.

Fleck, J. and D. K. Hofmann. 1990. The efficiency of metamorphosis inducing oligopeptides in Cassiopea species (Cnidaria: Scyphozoa) depends on both primary structure and aminoand carboxy terminal substituents. Verh Dtsch Zool Ges 83: 452-453.

Fleck, J. 1998. Chemical fate of a metamorphic inducer in larvaelike buds of the cnidarian Cassiopea andromeda. Biological Bulletin 194: 83-91.

Forskål, P. 1775. Desriptiones Animalium Avium, Amphibiorum, Piscium, Insectorum, Vermium; Quae in Intinere Orientali Observavit Petrus Forskål. Copenhagen: Niebuhr, Carsten.

Forskål, P. and C. Niebuhr. 1776. Icones rerum naturalium: quas in itinere orientali depingi. Copenhagen.

France, S. C. and L. L. Hoover. 2002. DNA sequences of the mitochondrial COI gene have low levels of divergence among deep-sea octocorals (Cnidaria: Anthozoa). Hydrobiologia 471: 149-155.

Freeman, C. J., E. W. Stoner, C. G. Easson, et al. 2016. Symbiont carbon and nitrogen assimilation in the CassiopeaSymbiodinium mutualism. Marine Ecology Progress Series 544: 281-286.

Freudenthal, H. D. 1959. Observations on the algal cells (zooxanthellae) inhabiting the anemone Cassiopea sp. J. Protozool Nr. 6: 12.

Freudenthal, H. D. 1962. Symbiodinium gen. nov. and Symbiodinium microadriaticum sp. nov., a Zooxanthella: Taxonomy, life cycle, and morphology. J. Protozool. 9: 45-52.

Fuller, Z. L., V. J. L. Mocellin, L. A. Morris, et al. 2020. Population genetics of the coral Acropora millepora: Toward genomic prediction of bleaching. Science 369: eaba4674.

Gamero-Mora, E., R. Halbauer, V. Bartsch, et al. 2019. Regenerative capacity of the upside-down jellyfish Cassiopea xamachana. Zoological Studies 58.

Garcia-Fernàndez, J. 2005. Hox, ParaHox, ProtoHox: Facts and guesses. Hereditary 94: 145-152.

Gauchat, D., F. Mazet, C. Berney, et al. 2000. Evolution of Antpclass genes and differential expression of Hydra Hox/paraHox genes in anterior patterning. Proceedings of the National Academy of Sciences of the United States of America 97: 4493-4498.

Gehrke, A. R., E. Neverett, Y. Luo, et al. 2019. Acoel genome reveals the regulatory landscape of whole-body regeneration. Science 363, Nr. 6432.

Gershwin, L. A., W. Zeidler and P. J. F. Davie. 2010. Medusae (Cnidaria) of Moreton Bay, Queensland, Australia. Memoirs of the Queensland Museum 54: 47-108.

Gilbert, S. F. 2016. Chapter twenty-two: Developmental plasticity and developmental symbiosis: The return of eco-devo. In Current topics in developmental biology, hg. von Paul M. Wassarman, 116: 415-433. Essays on Developmental Biology, Part A. Academic Press, 1.

Gilbert, S. F. and D. Epel. 2015. Ecological developmental biology: The environmental regulation of development, health, and evolution. 2nd ed. Sunderland, MA, USA: Sinauer Associates is an imprint of Oxford University Press.

Gohar, H. A. F. and A. M. Eisawy. 1960a. The biology of Cassiopea andromeda (from the Red Sea) (with a note on the species 
problem). Publications of the Marine Biological Station, Ghardaqa 11: 3-39.

Gohar, H. A. F. and M. Eisawy. 1960b. The development of Cassiopea andromeda (Scyphomedusae). Publs Marine Biology Station Ghardaqa 11: 148-190.

Gold, D. A., T. Katsuki, Y. L., Xifeng, et al. 2019. The genome of the jellyfish Aurelia and the evolution of animal complexity. Nature Ecology and Evolution 3: 96-104.

Goldfarb, A. J. 1914. Changes in salinity and their effects upon the regeneration of Cassiopea xamachana. Papers from the Tortugas Laboratory of the Carnegie Institution 6: 85-94.

Gómez-Daglio, L. and M. N. Dawson. 2017. Species richness of jellyfishes (Scyphozoa: Discomedusae) in the tropical Eastern Pacific: Missed taxa, molecules, and morphology match in a biodiversity hotspot. Invertebrate Systematics 31: 635-663.

Grant, A. J., D. A. Trautman, S. Frankland, et al. 2003. A symbiosome membrane is not required for the actions of two host signalling compounds regulating photosynthesis in symbiotic algae isolated from cnidarians. Comparative Biochemistry and Physiology: A Molecular and Integrative Physiology 135: 337-345.

Haeckel, E. 1880. System der Acraspeden-Zweite Hälfte des Systems der Medusen. Denkschriften der MedizinischNaturwissenschaftlichen Gesellschaft zu Jena: 82.

Hamlet, C., A. Santhanakrishnan and L. A. Miller. 2011. A numerical study of the effects of bell pulsation dynamics and oral arms on the exchange currents generated by the upside-down jellyfish Cassiopea xamachana. Journal of Experimental Biology 214: 1911-1921.

Harrison, P. L., R. C. Babcock, G. D. Bull, et al. 1984. Mass spawning in tropical reef corals. Science 223: 1186-1189.

Hartmann, A. C., K. L. Marhaver, A. Klueter, et al. 2019. Acquisition of obligate mutualist symbionts during the larval stage is not beneficial for a coral host. Molecular Ecology 28: 141-155.

He, S., F. Del Viso, C. Y. Chen, et al. 2018. An axial Hox code controls tissue segmentation and body patterning in Nematostella vectensis. Science 361: 1377-1380.

Hebert, P. D. N., S. Ratnasingham and J. R. DeWaard. 2003. Barcoding animal life: Cytochrome c oxidase subunit 1 divergences among closely related species. Proceedings of the Royal Society B: Biological Sciences 270: S96-S99.

Heins, A., T. Glatzel and S. Holst. 2015. Revised descriptions of the nematocysts and the asexual reproduction modes of the scyphozoan jellyfish Cassiopea andromeda (Forskål, 1775). Zoomorphology 134: 351-366.

Hofmann, D. K. and M. Gottlieb. 1991. Bud formation in the scyphozoan Cassiopea andromeda: Epithelial dynamics and fate map. Hydrobiologia 216: 53-59.

Hofmann, D. K. and M. G. Hadfield. 2002. Hermaphroditism, gonochorism, and asexual reproduction in Cassiopea sp.: An immigrant in the islands of Hawai'i. Invertebrate Reproduction and Development 41: 215-221.

Hofmann, D. K. and T. G. Honegger. 1990. Bud formation and metamorphosis in Cassiopea andromeda (Cnidaria: Scyphozoa): A developmental and ultrastructural study. Marine Biology 105: 509-518.

Hofmann, D. K. and B. P. Kremer. 1981. Carbon metabolism and strobilation in Cassiopea andromedea (Cnidaria: Scyphozoa): Significance of endosymbiotic dinoflagellates. Marine Biology 65: 25-33.

Hofmann, D. K., R. Neumann and K. Henne. 1978. Strobilation, budding and initiation of scyphistoma morphogenesis in the Rhizostome Cassiopea andromeda (Cnidaria: Scyphozoa). Marine Biology 47: 161-176.
Hofmann, D. K. and U. Brandt. 1987. Induction of metamorphosis in the symbiotic scyphozoan Cassiopea andromeda: Role of marine bacteria and of biochemicals. Symbiosis 4: 99-116.

Hofmann, D. K., W. K. Fitt and J. Fleck. 1996. Checkpoints in the life-cycle of Cassiopea spp.: Control of metagenesis and metamorphosis in a tropical jellyfish. International Journal of Developmental Biology 40: 331-338.

Hofmann, D. K. and G. Henning. 1991. Effects of axenic culture conditions on asexual reproduction and metamorphosis in the symbiotic scyphozoan Cassiopea andromeda. Symbiosis 10: 83-93.

Holland, B. S., M. N. Dawson, G. L. Crow, et al. 2004. Global phylogeography of Cassiopea (Scyphozoa: Rhizostomeae): Molecular evidence for cryptic species and multiple invasions of the Hawaiian Islands. Marine Biology 145: 1119-1128.

Hu, M., X. Zheng, C. M. Fan, et al. 2020. Lineage dynamics of the endosymbiotic cell type in the soft coral Xenia. Nature 582: 534-538.

Hui, J. H. L., P. W. H. Holland and D. E. K. Ferrier. 2008. Do cnidarians have a ParaHox cluster? Analysis of synteny around a Nematostella homeobox gene cluster. Evolution and Development 10: 725-730.

Hummelinck, P. Wagenaar. 1968. Caribbean scyphomedusae of the genus Cassiopea. Studies on the Fauna of Curaçao and other Caribbean Islands 25: 1-57.

Jantzen, C., C. Wild, M. Rasheed, et al. 2010. Enhanced pore-water nutrient fluxes by the upside-down jellyfish Cassiopea sp. in a Red Sea coral reef. Marine Ecology Progress Series 411: $117-125$.

Jarms, G. and A. C. Morandini. 2019. World Atlas of Jellyfish. Dölling and Galitz Verlag.

Jensch, F. and D. K. Hofmann. 1997. The cnidomes of Cassiopea andromeda Forskål, 1775, and Cassiopea xamachana Bigelow, 1882 (Cnidaria: Scyphozoa). Proceedings of the 6th International Conference on Coelenterate Biology, 279-285.

Jinek, M., K. Chylinski, I. Fonfara, et al. 2012. A programmable dual-RNA-guided DNA endonuclease in adaptive bacterial immunity. Science 337, Nr. 6096.

Jones, V. A. S., M. Bucher, E. A. Hambleton et al. 2018. Microinjection to deliver protein, mRNA, and DNA into zygotes of the cnidarian endosymbiosis model Aiptasia sp. Scientific Reports 8: 16437.

Kamm, K. and B. Schierwater. 2007. Ancient linkage of a POU class 6 and an anterior Hox-like gene in Cnidaria: Implications for the evolution of homeobox genes. Journal of Experimental Zoology Part B: Molecular and Developmental Evolution 308B: 777-784.

Kayal, E., B. Bentlage, A. G. Collins, et al. 2012. Evolution of linear mitochondrial genomes in medusozoan cnidarians. Genome Biology and Evolution 4: 1-12.

Kayal, E., B. Bentlage, M. S. Pankey, et al. 2018. Phylogenomics provides a robust topology of the major cnidarian lineages and insights on the origins of key organismal traits. BMC Evolutionary Biology 18, Nr. 68.

Keable, S. J. and S. T. Ahyong. 2016. First records of the invasive "upside-down jellyfish", Cassiopea (Cnidaria: Scyphozoa: Rhizostomeae: Cassiopeidae), from coastal lakes of New South Wales, Australia. Records of the Australian Museum 68: 23-30.

Khabibulina, V. and V. Starunov. 2019. Musculature development in planuloids of Cassiopeia xamachana (Cnidaria: Scyphozoa). Zoomorphology 138: 297-306.

Khalturin, K., C. Shinzato, M. Khalturina, et al. 2019. Medusozoan genomes inform the evolution of the jellyfish body plan. Nature Ecology and Evolution 3: 811-822. 
Kim, H. M., J. A. Weber, N. Lee, et al. 2019. The genome of the giant Nomura's jellyfish sheds light on the early evolution of active predation. BMC Biology 17: 28.

King, J. M. 1980. Direct fission: An undescribed reproductive method in hydromedusae. Bulletin of Marine Science 30: 522-525.

Klein, S. G., K. A. Pitt, C. H. Lucas, et al. 2019. Night-time temperature reprieves enhance the thermal tolerance of a symbiotic cnidarian. Frontiers in Marine Science 6, Nr. July: 453.

Kraus, Y. A. and A. V. Markov. 2016. The gastrulation in Cnidaria: A key to understanding phylogeny or the chaos of secondary modifications? Zhurnal Obshchě Biologii 77: 83-105.

Kuhn, K., B. Streit and Bernd Schierwater. 1999. Isolation of Hox genes from the Scyphozoan Cassiopeia xamachana: Implications for the early evolution of Hox genes. Journal of Experimental Zoology 285: 63-75.

Kvennefors, E. C. E., W. Leggat, O. Hoegh-Guldberg, et al. 2008. An ancient and variable mannose-binding lectin from the coral Acropora millepora binds both pathogens and symbionts. Developmental and Comparative Immunology 32: 1582-1592.

LaJeunesse, T. C. 2017. Validation and description of Symbiodinium microadriaticum, the type species of Symbiodinium (Dinophyta). Journal of Phycology 53: 1109-1114.

LaJeunesse, T. C. 2020. Zooxanthellae. Current Biology 30: R1110-R1113.

LaJeunesse, T. C., J. E. Parkinson, P. W. Gabrielson, et al. 2018. Systematic revision of Symbiodiniaceae highlights the antiquity and diversity of coral endosymbionts. Current Biology 28: 2570-2580.

LaJeunesse, T. C., D. T. Pettay, E. M. Sampayo, et al. 2010. Longstanding environmental conditions, geographic isolation and host-symbiont specificity influence the relative ecological dominance and genetic diversification of coral endosymbionts in the genus Symbiodinium. Journal of Biogeography 37: 785-800.

Lampert, K. P. 2016. Cassiopea and its zooxanthellae. In: The Cnidaria, past, present and future: The world of Medusa and her sisters, Stefano Goffredo and Zvy Dubinsky (Eds.). Springer International Publishing.

Lampert, K. P., P. Bürger, S. Striewski, et al. 2012. Lack of association between color morphs of the jellyfish Cassiopea andromeda and zooxanthella clade. Marine Ecology 33: 364-369.

Lehnert, E. M., M. E. Mouchka, M. S. Burriesci, et al. 2014. Extensive differences in gene expression between symbiotic and aposymbiotic cnidarians. G3: Genes, Genomes, Genetics 4: 277-295.

Li, Qiao, H. Y. and T. P. Zhong. 2015. Regeneration across metazoan phylogeny: Lessons from model organisms. Journal of Genetics and Genomics 42: 57-70.

Li, Yunfeng, L. G., Y. Pan, M. Tian, et al. 2020. Chromosome-level reference genome of the jellyfish Rhopilema esculentum. GigaScience 9, Nr. 4.

Lieshout, J., S. V. and V. J. Martin. 1992. Development of planuloid buds of Cassiopea xamachana (Cnidaria: Scyphozoa). Transactions of the American Microscopical Society 111: 89-110.

Light, S. F. 1914. Some Philippine Scyphomedusae, including two new genera, five new species, and one new variety. Philippine Journal of Science 9: 195-231.

Light, S. F. 1924. A new species of Scyphomedusan jellyfish in Chinese waters. The China Journal of Science and Arts 2: $449-450$.
Ludwig, F. D. 1969. Die Zooxanthellen bei Cassiopea andromeda Eschscholtz 1829 (Polyp-Stadium) und ihre Bedeutung für die Strobilation. Zoologische Jahrbücher. Abteilung für Anatomie und Ontogenie der Tiere Abteilung für Anatomie und Ontogenie der Tiere 86: 238-277.

Lukyanov, K. A. and V. V. Belousov. 2014. Genetically encoded fluorescent redox sensors. Biochimica et Biophysica Acta (BBA)—General Subjects 1840: 745-756.

Maas, O. 1903. Die Scyphomedusen der Siboga-Expedition. Siboga-expeditie 11. Leiden: Buchhandlung und druckerei vormals E. J. Brill.

Martin, V. J. and F. S. Chia. 1982. Fine structure of a scyphozoan planula, Cassiopeia xamachana. The Biological Bulletin 163 , Nr. 2.

Matthews, B. J. and L. B. Vosshall. 2020. How to turn an organism into a model organism in 10 "easy" steps. Journal of Experimental Biology 223: 223.

Mayer, A. G. 1908. Rhythmical pulsation of the medusae. 47. Aufl. Washington, DC.

Mayer, A. G. 1906. Medusae of the Hawaiian Islands collected by the steamer Albatross in 1902. Washington, DC: Government Printing Office.

Mayer, A. G. 1910. Medusae of the world: Volume III the scyphomedusae. Washington, DC: Carnegie Institution of Washington.

McFall-Ngai, M., M. G. Hadfield, T. C. G. Bosch, et al. 2013. Animals in a bacterial world, a new imperative for the life sciences. Proceedings of the National Academy of Sciences of the United States of America 110: 3229-3236.

McGill, C. J. and C. M. Pomory. 2008. Effects of bleaching and nutrient supplementation on wet weight in the jellyfish Cassiopea xamachana (Bigelow) (Cnidaria: Scyphozoa). Marine and Freshwater Behaviour and Physiology 41: 179-189.

McIlroy, S. E. and M. A. Coffroth. 2017. Coral ontogeny affects early symbiont acquisition in laboratory-reared recruits. Coral Reefs 36: 927-932.

Medina-Feliciano, J. G., S. Pirro, J. E. García-Arrarás, et al. 2020. Draft genome of the sea cucumber Holothuria glaberrima, a model for the study of regeneration. bioRxiv.

Mellas, R. E., S. E. McIlroy, W. K. Fitt et al. 2014. Variation in symbiont uptake in the early ontogeny of the upside-down jellyfish, Cassiopea spp. Journal of Experimental Marine Biology and Ecology 459: 38-44.

Mirsky, A. E. and H. Ris. 1951. The desoxyribonucleic acid content of animal cells and its evolutionary significance. The Journal of general physiology 34: 451-462.

Mohamed, A. R., V. Cumbo, S. Harii, et al. 2016. The transcriptomic response of the coral Acropora digitifera to a competent Symbiodinium strain: The symbiosome as an arrested early phagosome. Molecular Ecology 25: 3127-3141.

Morandini, A. C., D. Ascher, S. N. Stampar, et al. 2005. Cubozoa e Scyphozoa (Cnidaria: Medusozoa) de águas costeiras do Brasil. Iheringia. Série Zoologia 95: 281-294.

Morandini, A. C. and F. L. da Silveira. 2001. Sexual reproduction of Nausithoe aurea (Scyphozoa, Coronatae). Gametogenesis, egg release, embryonic development, and gastrulation. Scientia Marina 65: 139-149.

Morandini, A. C., S. N. Stampar, M. M. Maronna, et al. 2017. All non-indigenous species were introduced recently? The case study of Cassiopea (Cnidaria: Scyphozoa) in Brazilian waters. Journal of the Marine Biological Association of the United Kingdom 97: 321-328.

Mortillaro, J. M., K. A. Pitt, S. Y. Lee, et al. 2009. Light intensity influences the production and translocation of fatty acids 
by zooxanthellae in the jellyfish Cassiopea sp. Journal of Experimental Marine Biology and Ecology 378: 22-30.

Muscatine, L. and J. W. Porter. 1977. Reef Corals: Mutualistic symbioses adapted to nutrient-poor environments. BioScience 27: 454-460.

Nakai, J., M. Ohkura and K. Imoto. 2001. A high signal-to-noise $\mathrm{Ca} 2+$ probe composed of a single green fluorescent protein. Nature Biotechnology 19: 137-141.

Nakanishi, N., D. Yuan, D. K. Jacobs, et al. 2008. Early development, pattern, and reorganization of the planula nervous system in Aurelia (Cnidaria, Scyphozoa). Development Genes and Evolution 218: 511-524.

Nath, R. D., C. N. Bedbrook, M. J. Abrams, et al. 2017. The jellyfish Cassiopea exhibits a sleep-like state. Current Biology 27: 2984-2990.

Neumann, R. 1979. Bacterial induction of settlement and metamorphosis in the planula larvae of Cassiopea andromeda (Cnidaria: Scyphozoa, Rhizostomeae). Marine Ecology Progress Series 1, Nr. 1: 21-28.

Newkirk, C. R., T. K. Frazer, M. Q. Martindale, et al. 2020. Adaptation to bleaching: Are thermotolerant Symbiodiniaceae strains more successful than other strains under elevated temperatures in a model symbiotic cnidarian? Frontiers in Microbiology 11: 822.

Niggl, W., M. S. Naumann, U. Struck, et al. 2010. Organic matter release by the benthic upside-down jellyfish Cassiopea sp. fuels pelagic food webs in coral reefs. Journal of Experimental Marine Biology and Ecology 384: 99-106.

Ohdera, A., C. L. Ames, R. B. Dikow, et al. 2019. Box, stalked, and upside-down? Draft genomes from diverse jellyfish (Cnidaria, Acraspeda) lineages: Alatina alata (Cubozoa), Calvadosia cruxmelitensis (Staurozoa), and Cassiopea xamachana (Scyphozoa). GigaScience 8, Nr. 7.

Ohdera, A. H., M. J. Abrams, C. L. Ames et al. 2018. Upside-down but headed in the right direction: Review of the highly versatile Cassiopea xamachana system. Frontiers in Ecology and Evolution 6: 35.

Ohdera, A. H., K. Attarwala, H. Rubain, et al. In prep (a). Genomic insights of bacteria responsible for settlement and metamorphosis of Cassiopea xamachana larvae.

Ohdera, A. H., V. Avila-Magaña, V. Sharp et al. In prep (b). Modulation of gene expression driven by symbiosis: Strobilation mechanism in the upside-down jellyfish.

Ojimi, M. C. and M. Hidaka. 2010. Comparison of telomere length among different life cycle stages of the jellyfish Cassiopea andromeda. Marine Biology 157: 2279-2287.

Ojimi, M. C., N. Isomura and M. Hidaka. 2009. Telomerase activity is not related to life history stage in the jellyfish Cassiopea sp. Comparative Biochemistry and Physiology: A Molecular and Integrative Physiology 152: 240-244.

Pallas, Peter Simon. 1774. Spicilegia zoologica: quibus novae imprimis et obscurae animalium species iconibus, descriptionibus atque commentariis illustrantur. Hg. von August Lange. 10. Aufl. Berlin.

Passano, L. M. 2004. Spasm behavior and the diffuse nerve-net in Cassiopea xamachana (Scyphozoa: Coelenterata). In: Hydrobiologia, 530-531:91-96.

Perkins, H. F. 1908. Notes on the occurrence of Cassiopea xamachana and Polyclonia frondosa at the Tortugas. Papers from the Tortugas Laboratory of the Carnegie Institution 1.

Phelan, M. A., J. L. Matta, Y. M. Reyes, et al. 2006. Associations between metals and the blue mesogleal protein of Cassiopea xamachana. Marine Biology 149: 307-312.
Pierce, J. 2005. A system for mass culture of upside-down jellyfish Cassiopea spp. as a potential food item for medusivores in captivity. International Zoo Yearbook 39: 62-69.

Pinzón, J. H., B. Kamel, C. A. Burge, et al. 2015. Whole transcriptome analysis reveals changes in expression of immunerelated genes during and after bleaching in a reef-building coral. Royal Society Open Science 2: 140214.

Polteya, D. G. D. Zhinidarich and A. Lui. 1985. Observations of asexual reproduction and regeneration in Cassiopea (Scyphozoa, Coelenterata). Zoologicheski Zhurnal 64:172-180.

Radwan, F. F. Y., J. W. Burnett, D. A. Bloom, et al. 2001. A comparison of the toxinological characteristics of two Cassiopea and Aurelia species. Toxicon 39: 245-257.

Rahat, M. and O. Adar. 1980. Effect of symbiotic zooxanthellae and temperature on budding and strobilation in Cassiopeia andromeda (Eschscholz). Biological Bulletin 159: 394-401.

Reich, H. G., D. L. Robertson and G. Goodbody-Gringley. 2017. Do the shuffle: Changes in Symbiodinium consortia throughout juvenile coral development. PLoS One 12: e0171768.

Richardson, A. J., A. Bakun, G. C. Hays, et al. 2009. The jellyfish joyride: Causes, consequences and management responses to a more gelatinous future. Trends in Ecology \& Evolution 24: 312-322.

Rodriguez-Lanetty, M., W. S. Phillips and V. M. Weis. 2006. Transcriptome analysis of a cnidarian-dinoflagellate mutualism reveals complex modulation of host gene expression. BMC Genomics 7, Nr. 23.

Ruppert, E. E., R. S. Fox and R. D. Barnes. 2004. Invertebrate zoology: A functional evolutionary approach. Belmont, CA: Thomson-Brooks/Cole.

Sánchez-Alvarado, A. 2004. Regeneration and the need for simpler model organisms. Philosophical Transactions of the Royal Society of London. Series B: Biological Sciences 359: 759-763.

Santhanakrishnan, A., M. Dollinger, C. L. Hamlet, et al. 2012. Flow structure and transport characteristics of feeding and exchange currents generated by upside-down Cassiopea jellyfish. Journal of Experimental Biology 215: 2369-2381.

Schembri, P. J., A. Deidun and P. J. Vella. 2010. First record of Cassiopea andromeda (Scyphozoa: Rhizostomeae: Cassiopeidae) from the central Mediterranean Sea. Marine Biodiversity Records 3: e6.

Schierwater, B. and K. Kuhn. 1998. Homology of Hox genes and the Zootype concept in early metazoan evolution. Molecular Phylogenetics and Evolution 9: 375-381.

Schummer, M., I. Scheurlen, C. Schaller, et al. 1992. HOM/HOX homeobox genes are present in hydra (Chlorohydra viridissima) and are differentially expressed during regeneration. EMBO Journal 11: 1815-1823.

Schwarz, J. A., D. A. Krupp and V. M. Weis. 1999. Late larval development and onset of symbiosis in the scleractinian coral Fungia scutaria. The Biological Bulletin 196: 70-79.

Shao, Y., X. B. Wang, J. J. Zhang, et al. 2020. Genome and singlecell RNA-sequencing of the earthworm Eisenia andrei identifies cellular mechanisms underlying regeneration. Nature Communications 11: 2656.

Shearer, T. L., M. J. H. Van Oppen, S. L. Romano, et al. 2002. Slow mitochondrial DNA sequence evolution in the Anthozoa (Cnidaria). Molecular Ecology 11: 2475-2487.

Shikuma, N. J., I. Antoshechkin, J. M. Medeiros, et al. 2016. Stepwise metamorphosis of the tubeworm Hydroides elegans is mediated by a bacterial inducer and MAPK signaling. 
Proceedings of the National Academy of Sciences of the United States of America 113: 10097-10102.

Shinzato, C., E. Shoguchi, T. Kawashima, et al. 2011. Using the Acropora digitifera genome to understand coral responses to environmental change. Nature 476: 320-323.

Shumaker, A., H. M. Putnam, H. Qiu et al. 2019. Genome analysis of the rice coral Montipora capitata. Scientific Reports 9: 2571.

Smith, H. G. 1936. Contribution to the anatomy and physiology of Cassiopea frondosa. Papers from the Tortugas Laboratory of the Carnegie Institution $31 \mathrm{p}$.

Stephens, L. and D. Calder. 2006. Seafaring scientist: Alfred Goldsborough Mayor, pioneer in marine biology. University of South Caroline Press, Columbia.

Stiasny, G. 1924. Ueber einige von Dr. CJ van der Horst bei Curaçao gesammelte Medusen. Bijdragen tot de Dierkunde 23: 83-92.

Stockard, C. R. 1908. Studies of tissue growth. Archiv für Entwicklungsmechanik der Organismen 29: 15-32.

Stoner, E. W., C. A. Layman, et al. 2011. Effects of anthropogenic disturbance on the abundance and size of epibenthic jellyfish Cassiopea spp. Marine Pollution Bulletin 62: 1109-1114.

Szmant, A. M. 1986. Reproductive ecology of Caribbean reef corals. Coral Reefs 5: 43-53.

Thé, J., H. S. Barroso, M. Mammone, et al. 2020. Aquaculture facilities promote populational stability throughout seasons and increase medusae size for the invasive jellyfish Cassiopea andromeda. Marine Environmental Research: 105161.

Thieme, C. and D. K. Hofmann. 2003. An endogenous peptide is involved in internal control of metamorphosis in the marine invertebrate Cassiopea xamachana (Cnidaria: Scyphozoa). Development Genes and Evolution 213: 97-101.

Thompson, J. R., H. E. Rivera, C. J. Closek, et al. 2015. Microbes in the coral holobiont: Partners through evolution, development, and ecological interactions. Frontiers in Cellular and Infection Microbiology 4: 176.

Thornhill, D. J., M. W. Daniel, T. C. LaJeunesse, et al. 2006. Natural infections of aposymbiotic Cassiopea xamachana scyphistomae from environmental pools of Symbiodinium. Journal of Experimental Marine Biology and Ecology 338: 50-56.

Tiozzo, S. and R. R. Copley. 2015. Reconsidering regeneration in metazoans: An evo-devo approach. Frontiers in Ecology and Evolution 3: 67.

Todd, B. D., D. J. Thornhill and W. K. Fitt. 2006. Patterns of inorganic phosphate uptake in Cassiopea xamachana: A bioindicator species. Marine Pollution Bulletin 52: 515-521.

Tran, C. and M. G. Hadfield. 2011. Larvae of Pocillopora damicornis (Anthozoa) settle and metamorphose in response to surface-biofilm bacteria. Marine Ecology Progress Series 433: 85-96.

Trench, R. K. 1979. The cell biology of plant-animal symbiosis. Annual Review of Plant Physiology 30: 485-531.

Uchida, T. 1970. Occurrence of a rhizostome medusa, Cassiopea mertensii Brandt from the Hawaiian Islands. Annotat. Zool. Jap. 43: 102-104.
Van Etten, M. L., J. K. Conner, S. M. Chang, et al. 2017. Not all weeds are created equal: A database approach uncovers differences in the sexual system of native and introduced weeds. Ecology and Evolution 7: 2636-2642.

Van Woesik, R., F. Lacharmoise and S. Köksal. 2006. Annual cycles of solar insolation predict spawning times of Caribbean corals. Ecology Letters 9: 390-398.

Verde, E. A. and L. R. McCloskey. 1998. Production, respiration, and photophysiology of the mangrove jellyfish Cassiopea xamachana symbiotic with zooxanthellae: Effect of jellyfish size and season. Marine Ecology Progress Series 168: 147-162.

Vijayan, N., K. A. Lema, B. T. Nedved, et al. 2019. Microbiomes of the polychaete Hydroides elegans (Polychaeta: Serpulidae) across its life-history stages. Marine Biology 166: 19.

Voolstra, C. R., J. A. Schwarz, J. Schnetzer, et al. 2009. The host transcriptome remains unaltered during the establishment of coral-algal symbioses. Molecular Ecology 18: $1823-1833$.

Waddington, C. H. 1942. Canalization of development and the inheritance of acquired characters. Nature 150: 563-565.

Welsh, D. T., R. J. K. Dunn and T. Meziane. 2009. Oxygen and nutrient dynamics of the upside down jellyfish (Cassiopea sp.) and its influence on benthic nutrient exchanges and primary production. Hydrobiologia 635: 351-362.

Widmer, C. L. 2008. Life cycle of Chrysaora fuscescens (Cnidaria: Scyphozoa) and a key to sympatric ephyrae. Pacific Science 62: 71-82.

Winstead, D., A. Ohdera, M. Medina, et al. 2018. Symbiodinium proliferation inside a cnidarian host vessel are competitive and dynamic. SICB 2018 Annual Meeting Abstracts, 58: e1e264. San Francisco, CA, März.

Wolk, M., M. Rahat, W. K. Fitt, et al. 1985. Cholera toxin and thyrotropine can replace natural inducers required for the metamorphosis of larvae and buds of the scyphozoan Cassiopea andromeda. Wilhelm Roux's Archives of Developmental Biology 194: 487-490.

Yokoyama, J., N. Hanzawa, Y. Arai, et al. 2017. Phylogenetic relationships and morphological variations of upside-down jellyfishes, Cassiopea spp. inhabiting Palau Islands. Phylogenetic relationships and morphological variations of upsidedown jellyfishes, Cassiopea spp. inhabiting Palau Islands. Biogeography 10: 133-141.

Yonge, C. M. 1928-1929. Studies on the physiology of corals. IV: The structure, distribution and physiology of the zooxanthellae. Scientific Reports/Great Barrier Reef Expedition 1: 135-176.

Yonge, C.M., Nicholls, A.G. 1931. Studies on the physiology of corals. IV. The structure, distribution and physiology of the zooxanthellae. Sci. Rept. Gr. Barrier Reef Exped. 1928-1929. Brit. Mus. (Nat. Hist.) 1:135-176.

Yuan, D., N. Nakanishi, D. K. Jacobs, et al. 2008. Embryonic development and metamorphosis of the scyphozoan Aurelia. Development Genes and Evolution 218: 525-539. 